\title{
Quantization of Poisson manifolds from the integrability of the modular function
}

\author{
F. Bonechi*, N. Ciccoli ${ }^{\dagger}$, J. Qiu $\stackrel{\ddagger}{\ddagger}$ M. Tarlini ${ }^{\S}$
}

June 18, 2013

\begin{abstract}
We discuss a framework for quantizing a Poisson manifold via the quantization of its symplectic groupoid, that combines the tools of geometric quantization with the results of Renault's theory of groupoid $C^{*}$-algebras. This setting allows very singular polarizations. In particular we consider the case when the modular function is multiplicatively integrable, i.e. when the space of leaves of the polarization inherits a groupoid structure. If suitable regularity conditions are satisfied, then one can define the quantum algebra as the convolution algebra of the subgroupoid of leaves satisfying the Bohr-Sommerfeld conditions.

We apply this procedure to the case of a family of Poisson structures on $\mathbb{C} P_{n}$, seen as Poisson homogeneous spaces of the standard Poisson-Lie group $S U(n+1)$. We show that a bihamiltoniam system on $\mathbb{C} P_{n}$ defines a multiplicative integrable model on the symplectic groupoid; we compute the Bohr-Sommerfeld groupoid and show that it satisfies the needed properties for applying Renault theory. We recover and extend Sheu's description of quantum homogeneous spaces as groupoid $C^{*}$-algebras.
\end{abstract}

*INFN Sezione di Firenze, email: bonechi@fi.infn.it

†Dipartimento di Matematica, Univ. di Perugia, email: ciccoli@dipmat.unipg.it ¥Mathématiques, Université du Luxembourg, email: jian.qiu@uni.lu

§INFN Sezione di Firenze, email: marco.tarlini@fi.infn.it 


\section{Introduction}

The quantization of a Poisson manifold is well understood in terms of the deformation quantization of the algebra of smooth functions but is much more elusive if one looks for an operatorial quantization. In fact Kontsevich formula [15], together with the globalization procedure [6], gives an explicit formula for the deformation quantization of any smooth Poisson manifold. Nothing similar exists for the operatorial quantization, in whatever sense of the word.

The concept of symplectic groupoid was introduced in the mid eighties by Karasev and Weinstein as a tool for quantization of Poisson manifolds. Indeed, when the Poisson manifold $(M, \pi)$ is integrable there exists a unique symplectic manifold $\mathcal{G}(M, \pi)$, endowed with a compatible groupoid structure, that allows to reconstruct the Poisson tensor on its space of units $M$. The compatibility between the groupoid and symplectic structures is expressed by requiring that the graph of the multiplication is a lagrangian submanifold of $\overline{\mathcal{G}(M, \pi)} \times \overline{\mathcal{G}(M, \pi)} \times$ $\mathcal{G}(M, \pi)$, where $\overline{\mathcal{G}(M, \pi)}$ has the opposite symplectic structure. If $\mathcal{H}$ is the space of states obtained by means of some quantization procedure of $\mathcal{G}(M, \pi)$, then, by applying the semiclassical quantization dictionary, the graph of the multiplication is quantized by a vector in $\mathcal{H}^{*} \otimes \mathcal{H}^{*} \otimes \mathcal{H}$. This vector defines an algebra structure on $\mathcal{H}$, that should be considered as the algebra of quantum observables rather than the space of states.

The basic question that one has to address is what kind of quantization we are looking for. It seems a tall order to seek a map associating an operator to any classical observable, but rather one should look directly for the non commutative algebra of quantum observables. In fact, from the point of view of geometric quantization, such a map needs the quantization of any observable, while we know that this is out of reach even in favourable cases, since the polarization procedure selects a very narrow class of quantizable functions. Very recently, E. Hawkins in [14] proposed a general framework based on the geometric quantization of the symplectic groupoid where the quantization output is a $C^{*}$-algebra. Basically, the idea of [14] is to look for a multiplicative polarization $F \subset T_{\mathbb{C}} \mathcal{G}(M, \pi)$ that allows for the definition of a convolution product on the space of polarized sections. The best case is that of a real polarization defined by a Lie groupoid fibration $\mathcal{G}(M, \pi) \rightarrow \mathcal{G}_{F}(M, \pi)$ over some Lie groupoid $\mathcal{G}_{F}(M, \pi)$; the $C^{*}$-algebra in this case is the convolution algebra of the groupoid $\mathcal{G}_{F}(M, \pi)$ of lagrangian leaves. The usual problem of geometric quantization is to find such polarizations, in particular real ones very often do not exist.

Our starting observation is that thanks to the groupoid structure we can consider much more general polarizations than those needed by geometric quantization. In fact, suppose that, instead of a smooth polarization, we just have a topological quotient of groupoids $\mathcal{G}(M, \pi) \rightarrow \mathcal{G}_{F}(M, \pi)$ with lagrangian fibres. 
Let us call $\mathcal{G}_{F}^{b s}(M, \pi)$ the subgroupoid of Bohr-Sommerfeld (BS) leaves, i.e. those leaves with trivial holonomy. In order to define a convolution algebra it is enough that $\mathcal{G}_{F}^{b s}(M, \pi)$ admits a Haar system; this can be fulfilled in cases of polarizations that are very singular and hard to use in geometric quantization. In this case, quantization then follows from Renault theory on groupoid $C^{*}$-algebras [22] and fits in a transparent way with the Poisson geometry.

Indeed, given a Poisson manifold $(M, \pi)$, there are two cohomology classes in $H_{L P}^{\bullet}(M, \pi)$, the Lichnerowicz-Poisson cohomology, that are relevant for quantization. The Poisson tensor itself defines a class $[\pi] \in H_{L P}^{2}(M, \pi)$; when a prequantization of the symplectic groupoid is chosen, there is defined a 2-cocycle of the symplectic groupoid with values in $\mathbb{S}^{1}$ that we call the prequantization cocycle. This 2-cocycle can be 1 if and only if $[\pi]=0$. Moreover, for every volume form on $M$ it is defined the modular vector field that measures the non invariance of the volume form with respect to hamiltonian transformations. It defines a class in $H_{L P}^{1}(M, \pi)$, the modular class, independent on the choice of the volume form: it vanishes only if there exists an invariant volume form. The modular vector field is integrated to a 1-cocycle of the symplectic groupoid, that we call the modular function.

It is then natural to require that for our generalized polarization, both the modular function and the prequantization cocycle should descend to $\mathcal{G}_{F}(M, \pi)$; the general theory of groupoid $C^{*}$-algebras tells us how to define the convolution algebra, twisted by the prequantization cocycle; the modular function is quantized to an automorphism of the algebra: the modular operator in noncommutative geometry. Moreover it determines a quasi invariant measure (equivalently the KMS state); finally we get an Hilbert algebra structure (compatible scalar product and convolution) which corresponds to the algebra on the spaces of states that we are looking for.

Integrable models on the symplectic groupoid are a possible source for such singular polarizations and appear very naturally when the Poisson structure is not unimodular. We say that the modular function is multiplicatively integrable if there exists a maximal family of independent hamiltonians in involution with it such that the space of contour levels inherits the groupoid structure.

A class of examples where such construction is possible is given by compact Poisson-Lie groups and their homogeneous spaces. They are the semiclassical limit of quantum groups and their quantum homogeneous spaces. A series of papers by A.J. Sheu [25, 26] relates the quantum spaces to groupoid $C^{*}$-algebras. In certain cases (for instance the standard $\mathbb{C} P_{n, q, 0}$ and the odd dimensional spheres $\mathbb{S}_{q}^{2 n+1}$ ) he showed that the $C^{*}$-algebra is a groupoid $C^{*}$-algebra; for $S U_{q}(n)$ or in the non standard structures $\mathbb{C} P_{n, q, t}$, for $t \in(0,1)$, his characterization of the $C^{*}$-algebra is just as a subalgebra of a groupoid $C^{*}$-algebra. His analysis is based on representation theory of the quantum algebras. 
We conjecture that we can interpret the groupoids appearing in Sheu's papers as BS-leaves of some polarization of the symplectic groupoid integrating the semiclassical structure. Since they are in general non unimodular Poisson manifolds, we conjecture that these BS groupoids appear from the multiplicative integrability of the modular function.

In this paper we are going to show that this is actually true for the whole family of Poisson structures on $\mathbb{C} P_{n}$, thus recovering Sheu's description for the standard case and extending it in the non standard one. Moreover, as a byproduct of the construction we get the quantization of a class of Poisson submanifolds; in particular we recover the description of the odd dimensional spheres.

The simplest example is the so called Podles̀ two sphere and has been already studied in [2] (the integrability issues were not explicit there, for this point of view see the discussion in [3]).

In the general case, the relevant integrable model depends on a bihamiltonian structure existing on all compact hermitian spaces. In fact it was shown in [16] that on compact hermitian symmetric spaces there exists a couple of compatible Poisson structures $\left(\pi_{0}, \pi_{s}\right)$ defining a bihamiltonian system. The first one $\pi_{0}$, the Bruhat or standard Poisson structure, is the quotient of the compact Poisson-Lie group by a Poisson subgroup and $\pi_{s}$ is the inverse of the Kirillov symplectic form, once that we realize the symmetric space as a coadjoint orbit. By taking a linear combination we get a Poisson pencil, i.e. a family of Poisson homogeneous spaces $\pi_{t}=\pi_{0}+t \pi_{s}$. For $t=(0,1) \pi_{t}$ is called non standard. Since one of two Poisson structures is symplectic, we can define a maximal set of hamiltonians that are in involution with respect to every Poisson structure of the pencil (for a short account of this see [13]).

We analyze here the case of complex projective spaces and we are interested in the case where $t \in[0,1]$, when $\pi_{t}$ is degenerate. The integrable model corresponds to the toric structure of $\mathbb{C} P_{n}$ given by the action of the Cartan $\mathbb{T}^{n} \subset S U(n+1)$ and was studied in connection with the bihamiltonian structure in [13. The action of the Cartan on $\mathbb{C} P_{n}$ is hamiltonian in the symplectic case with momentum map $c: \mathbb{C} P_{n} \rightarrow \mathfrak{t}_{n}^{*}, \mathfrak{t}_{n}=$ Lie $^{n}$, and simply Poisson with respect to the Poisson structure $\pi_{t}$ for $t \in[0,1]$. This means that the action lifts to an hamiltonian action of $\mathbb{T}^{n}$ on the symplectic groupoid $\mathcal{G}\left(\mathbb{C} P_{n}, \pi_{t}\right)$ with momentum map $h: \mathcal{G}\left(\mathbb{C} P_{n}, \pi_{t}\right) \rightarrow \mathfrak{t}_{n}^{*}$. The couple $(c, h)$, where $c$ is pulled back to $\mathcal{G}\left(\mathbb{C} P_{n}, \pi_{t}\right)$ with the source map, defines an integrable model. We show in Proposition 6.1 that it is multiplicative. The modular vector field with respect to the FubiniStudy volume form is just the fundamental vector field of a particular element of $\mathfrak{t}_{n}$ so that the modular function is in involution with $(c, h)$.

Our main result is Theorem 6.3 where we give an explicit description of the Bohr-Sommerfeld groupoid $\mathcal{G}_{F}^{b s}\left(\mathbb{C} P_{n},, \pi_{t}\right)$ and show that it has a unique Haar system. Moreover, in Proposition 5.4 we show that the maximal Poisson sub- 
manifolds, including odd spheres, are quantized to subgroupoids of this BSgroupoid, showing the covariant nature of the correspondence. We show that the BS-groupoid for $t=0$ and the Poisson submanifold $\left(\mathbb{S}^{2 n-1}, \pi_{t}\right) \subset\left(\mathbb{C} P_{N}, \pi_{t}\right)$ are the same appearing in Sheu's description of $\mathbb{C} P_{n, q, 0}$ and $\mathbb{S}_{q}^{2 n-1}$; the result for $t=(0,1)$ conjecturally improves his description for $\mathbb{C} P_{n, q, t}$.

This is the plan of the paper. In Section 2 we give a very brief review of basic facts on integration of Poisson homogeneous spaces and groupoid $C^{*}$-algebras. In Section 3 we introduce the definition of multiplicative integrability of the modular function and the general quantization scheme. In Section 4 we describe the geometry of $\left(\mathbb{C} P_{n}, \pi_{t}\right)$ as Poisson homogeneous space of $S U(n+1)$ equipped with the quasitriangular Poisson-Lie structure. In Section 5 we interpret this family of Poisson structures as a Poisson pencil arising by the bihamiltonian system. In Section 6 we discuss the integrable model on the symplectic groupoid of $\left(\mathbb{C} P_{n}, \pi_{t}\right)$; we compute the Bohr-Sommerfeld leaves, discuss the Haar system and the quasi invariant measure associated to the modular function; we discuss the quantization of maximal Poisson submanifolds. Finally in Section 7 we relate our results to Sheu's description of quantum homogeneous spaces in terms of groupoid $C^{*}$-algebras.

Notations. We will denote a groupoid with $\mathcal{G}=\left(\mathcal{G}, \mathcal{G}_{0}, l_{\mathcal{G}}, r_{\mathcal{G}}, m_{\mathcal{G}}, \iota_{\mathcal{G}}, \epsilon_{\mathcal{G}}\right) ; \mathcal{G}_{0}$ is the space of units, $l_{\mathcal{G}}, r_{\mathcal{G}}: \mathcal{G} \rightarrow \mathcal{G}_{0}$ are the source and target maps, $\mathcal{G}_{2} \subset \mathcal{G} \times \mathcal{G}$ denotes those elements $\left(\gamma_{1}, \gamma_{2}\right) \in \mathcal{G}_{2}$ such that $r_{\mathcal{G}}\left(\gamma_{1}\right)=l_{\mathcal{G}}\left(\gamma_{2}\right)$. Moreover, $m_{\mathcal{G}}$ : $\mathcal{G}_{2} \rightarrow \mathcal{G}$ is the multiplication, $\iota_{\mathcal{G}}: \mathcal{G} \rightarrow \mathcal{G}$ is the inversion and $\epsilon_{\mathcal{G}}: \mathcal{G}_{0} \rightarrow \mathcal{G}$ is the embedding of units. We denote with ${ }_{x} \mathcal{G}=l_{\mathcal{G}}^{-1}(x)$ and $\mathcal{G}_{x}=r_{\mathcal{G}}^{-1}(x)$ for $x \in \mathcal{G}_{0}$. We say that $\mathcal{G}$ is source simply connected (Ssc) if ${ }_{x} \mathcal{G}$ is connected and simply connected for any $x \in \mathcal{G}_{0}$.

We denote with $\mathcal{G}_{k}$ the space of strings of $k$-composable elements of $\mathcal{G}$, with the convention that $\mathcal{G}_{1}=\mathcal{G}$. The face maps are $d_{i}: \mathcal{G}_{s} \rightarrow \mathcal{G}_{s-1}, i=0, \ldots s$, defined for $s>1$ as

$$
d_{i}\left(\gamma_{1}, \ldots \gamma_{s}\right)= \begin{cases}\left(\gamma_{2}, \ldots \gamma_{s}\right) & i=0 \\ \left(\gamma_{1}, \ldots \gamma_{i} \gamma_{i+1} \ldots\right) & 0<i<s \\ \left(\gamma_{1}, \ldots \gamma_{s-1}\right) & i=s\end{cases}
$$

and for $s=1$ as $d_{0}(\gamma)=l_{\mathcal{G}}(\gamma), d_{1}(\gamma)=r_{\mathcal{G}}(\gamma)$. The simplicial coboundary operator $\partial^{*}: \Omega^{k}\left(\mathcal{G}_{s}\right) \rightarrow \Omega^{k}\left(\mathcal{G}_{s+1}\right)$ is defined as

$$
\partial^{*}(\omega)=\sum_{i=0}^{s}(-)^{i} d_{i}^{*}(\omega)
$$

and $\partial^{* 2}=0$. The cohomology of this complex for $k=0$ is the real valued groupoid cohomology; s-cocycles are denoted as $Z^{s}(\mathcal{G}, \mathbb{R})$. 
If $\Lambda$ is a line bundle on $\mathcal{G}$, then $\partial^{*} \Lambda$ denotes the line bundle $d_{0}^{*} \Lambda \otimes d_{1}^{*} \Lambda^{*} \otimes d_{2}^{*} \Lambda$ over $\mathcal{G}_{2}$, where $d_{i}: \mathcal{G}_{2} \rightarrow \mathcal{G}$ denote the face maps defined in (1).

If $S \subset \mathcal{G}_{0}$ then we denote with $\mathcal{G}_{S}=l_{\mathcal{G}}^{-1}(S) \cap r_{\mathcal{G}}^{-1}(S)$ the subgroupoid of $\mathcal{G}$ obtained by restriction to $S$. If a group $G$ acts on $X$, the action groupoid $X \rtimes G$ is defined on $X \times G$ with structure maps as $l(x, g)=x, r(x, g)=x g$, $m\left[(x, g)\left(x g, g^{\prime}\right)\right]=\left(x, g g^{\prime}\right), \epsilon(x)=(x, e), \iota(x, g)=\left(x g, g^{-1}\right)$, for $x \in X$ and $g, g^{\prime} \in G$.

A Poisson structure is denoted $(M, \pi)$, where $\pi$ is the two tensor defining the Poisson bracket on the smooth manifold $M$. We denote with $\pi$ also the bundle map $\pi: T^{*} M \rightarrow T M$. The Lichnerowicz-Poisson differential is denoted with $d_{L P}(X)=[\pi, X]$; its cohomology with $H_{L P}(M, \pi)$.

\section{Poisson manifolds, symplectic groupoids and Haar systems}

We recall in this Section the background material that we will need later. In particular we introduce basic definitions and properties of Poisson manifolds and symplectic groupoids, of Poisson-Lie groups and their homogeneous spaces. Finally we discuss Haar systems on topological groupoids.

\subsection{Poisson manifolds and symplectic groupoids}

We say that a topological groupoid $\mathcal{G}$ is a Lie groupoid if $\mathcal{G}$ and $\mathcal{G}_{0}$ are smooth manifolds, all maps are smooth and $l_{\mathcal{G}}, r_{\mathcal{G}}$ are surjective submersions. A symplectic groupoid is a Lie groupoid, equipped with a symplectic form $\Omega_{\mathcal{G}}$, such that the graph of the multiplication is a lagrangian submanifold of $\overline{\mathcal{G}} \times \overline{\mathcal{G}} \times \mathcal{G}$, where $\overline{\mathcal{G}}$ means $\mathcal{G}$ with the opposite symplectic structure. There exists a unique Poisson structure on $\mathcal{G}_{0}$ such that $l_{\mathcal{G}}$ and $r_{\mathcal{G}}$ are Poisson and anti-Poisson morphisms respectively; the Poisson manifold $\mathcal{G}_{0}$, in such case, is said to be integrable. Given a Poisson manifold $(M, \pi)$ is always possible to define a (ssc) topological groupoid $\mathcal{G}(M, \pi)$ as the quotient of the space of cotangent paths with respect to cotangent homotopies. A cotangent path is a bundle map $(X, \eta): T[0,1] \rightarrow T^{*} M$ satisfying $d X+\pi(\eta)=0$; see [9, 5] for the definitions of cotangent homotopies and the groupoid structure maps. The obstruction for the existence of the smooth structure making $\mathcal{G}(M, \pi)$ to be the (ssc) symplectic groupoid integrating $(M, \pi)$ has been studied in [10].

Let $(M, \pi)$ be a Poisson manifold. A vector field $\chi \in \operatorname{Vect}(M)$ satisfying $d_{L P}(\chi)=0$ is called a Poisson vector field. Two distinguished classes are relevant for what follows. The first one is the class $[\pi] \in H_{L P}^{2}(M, \pi)$ defined by the Poisson 
tensor itself. Let us assume that $M$ is orientable, and let us choose a volume form $V_{M}$ on $M$. The modular vector field $\chi_{V_{M}}=\operatorname{div}_{V_{M}} \pi$ is Poisson; its cohomology class $\chi_{V_{M}} \in H_{L P}^{1}(M, \pi)$ does not depend on the choice of the volume form and is called the modular class.

Let $(M, \pi)$ be integrable so that $\mathcal{G}(M, \pi)$ is the ssc symplectic groupoid integrating it. Every Poisson vector field $\chi \in \operatorname{Vect}(M)$ can be integrated to a unique real valued groupoid 1-cocycle $f_{\chi} \in Z^{1}(\mathcal{G}(M, \pi), \mathbb{R})$ with the formula

$$
f_{\chi}[X, \eta]=\int_{0}^{1}\langle\eta(t), \chi(X(t))\rangle d t
$$

We call $f_{\chi_{V_{M}}}$ the modular function.

\subsection{Integration of Poisson homogeneous spaces}

A Poisson-Lie group is a Lie group $G$ endowed with a Poisson structure $\pi_{G}$ such that the group multiplication $\left(G \times G, \pi_{G} \oplus \pi_{G}\right) \rightarrow\left(G, \pi_{G}\right)$ is a Poisson map. This means that for each $g_{1}, g_{2} \in G$ we have that

$$
\pi_{G}\left(g_{1} g_{2}\right)=l_{g_{1 *}} \pi_{G}\left(g_{2}\right)+r_{g_{2} *} \pi_{G}\left(g_{1}\right) .
$$

As a consequence, there exists the simply connected dual Poisson-Lie group $\left(G^{*}, \pi_{G^{*}}\right)$, that acts infinitesimally on $G$, the so called dressing action. We assume that this action is integrated to a group action ( $\mathrm{G}$ is called complete); as a consequence also $G^{*}$ is complete. If $g \in G$ and $\gamma \in G^{*}$ we denote the left and right action of $G^{*}$ on $G$ as ${ }^{\gamma} g$ and $g^{\gamma}$, respectively; analogously the left and right actions of $G$ on $G^{*}$ are denoted as ${ }^{g} \gamma$ and $\gamma^{g}$, respectively.

Any Poisson-Lie group is integrable and its symplectic groupoid has been first described in [18]. If $G$ is complete then $\mathcal{G}\left(G, \pi_{G}\right)=G \times G^{*}$ with $l(g, \gamma)=g$, $r(g, \gamma)=g^{\gamma}, m\left(g_{1}, \gamma_{1}\right)\left(g_{1}^{\gamma_{1}}, \gamma_{2}\right)=\left(g_{1}, \gamma_{1} \gamma_{2}\right), \epsilon(g)=(g, e), \iota(g, \gamma)=\left(g^{\gamma}, \gamma^{-1}\right)$. The non degenerate Poisson tensor reads

$$
\pi_{\mathcal{G}}=\pi_{G}+\pi_{G^{*}}+\sum_{a} \ell_{T_{a}} r_{T^{a}}
$$

where $\ell_{T_{a}}$ and $r_{T^{a}}$ are left and right vector fields on $G$ and on $G^{*}$ with respect to a choice of dual basis of LieG and $\mathrm{Lie}^{*}$.

The (right) action of $\left(G, \pi_{G}\right)$ on $(M, \pi)$ is Poisson if the action seen as a map $\left(M \times G, \pi \oplus \pi_{G}\right) \rightarrow(M, \pi)$ is Poisson. This means that for each $m \in M$ and $g \in G$ we have that

$$
\pi(m g)=g_{*} \pi(m)+m_{*} \pi_{G}(g)
$$

If the action is transitive then $(M, \pi)$ is called a Poisson homogeneous space. If there exists $m \in M$ where $\pi(m)=0$ then one can show that the stability 
subgroup $H_{m} \subset G$ is coisotropic and viceversa if a closed subgroup $H \subset G$ is coisotropic then there exists a unique Poisson structure $\pi_{H \backslash G}$ on $H \backslash G$ such that the projection map $\mathrm{pr}_{H}$ is Poisson. We call these homogeneous spaces embeddable. We know that $(\mathrm{LieH})^{\perp} \subset(\mathrm{LieG})^{*}=\mathrm{Lie}^{*}$ is a Lie subalgebra; let us denote with $H^{\perp} \subset G^{*}$ the connected subgroup integrating (LieH) ${ }^{\perp}$, which we assume to be closed. Since $H^{\perp}$ is coisotropic then there exists a canonical Poisson structure $\pi_{G^{*} / H^{\perp}}$ on $G^{*} / H^{\perp}$; let $\operatorname{pr}_{\mathrm{H}^{\perp}}$ denote the projection map. Moreover, the dressing action of $H$ preserves $H^{\perp}$, and viceversa.

Let us recall here how the integration of embeddable homogeneous spaces is done in [1]. The left $G$ action on itself can be lifted to a symplectic action of $\mathcal{G}\left(G, \pi_{G}\right)$; this action admits a Lu momentum map $J: \mathcal{G}\left(G, \pi_{G}\right) \rightarrow G^{*}$, $J(g, \gamma)={ }^{g} \gamma$. If we denote $J_{H}=\operatorname{pr}_{H^{\perp}} \circ J$, then the symplectic groupoid integrating $\left(H \backslash G, \pi_{H \backslash G}\right)$ is the symplectic reduction $H \backslash J_{H}^{-1}\left(\operatorname{pr}_{H^{\perp}}(e)\right)$. The symplectic groupoid structure descends from $\mathcal{G}\left(G, \pi_{G}\right)$. We then get the following description

$$
\mathcal{G}\left(H \backslash G, \pi_{H \backslash G}\right)=\left\{\left(\operatorname{pr}_{H}(g), \gamma\right), g \in G,{ }^{g} \gamma \in H^{\perp}\right\} .
$$

Let us denote with $G_{H} \times_{H} H^{\perp}$ the fibre bundle associated to the homogeneous principal bundle with the dressing action of $H$ on $H^{\perp}$. The map $L: \mathcal{G}\left(H \backslash G, \pi_{H \backslash G}\right) \rightarrow G_{H} \times_{H} H^{\perp}$ defined as $L\left(\operatorname{pr}_{H}(g), \gamma\right)=\left[g,{ }^{g} \gamma\right]$ is a diffeomorphism commuting $l$ with the bundle projection; as a consequence $l$ is a fibration. By taking $L \circ \iota$ we prove the same result for the target map $r$.

\subsection{Haar systems}

Let us recall basic facts from [22] (see also the very brief description given in Section 4 of [2]). Let $\mathcal{T}$ be a topological groupoid and let $C_{c}(\mathcal{T})$ denote the space of continuous functions with compact support. A left Haar system for $\mathcal{T}$ is a family of measures $\left\{{ }_{x} \lambda, x \in \mathcal{T}_{0}\right\}$ on $\mathcal{T}$ such that

i) the support of ${ }_{x} \lambda$ is ${ }_{x} \mathcal{T}=l_{\mathcal{T}}^{-1}(x)$;

ii) for any $f \in C_{c}(\mathcal{T})$, and $x \in \mathcal{T}_{0}, \lambda(f)(x)=\int_{\mathcal{T}} f d_{x} \lambda$, defines $\lambda(f) \in C_{c}\left(\mathcal{T}_{0}\right)$;

iii) for any $\gamma \in \mathcal{T}$ and $f \in C_{c}(\mathcal{T}), \int_{\mathcal{T}} f\left(\gamma \gamma^{\prime}\right) d_{r_{\mathcal{T}}(\gamma)} \lambda\left(\gamma^{\prime}\right)=\int_{\mathcal{T}} f\left(\gamma^{\prime}\right) d_{l_{\mathcal{T}}(\gamma)} \lambda\left(\gamma^{\prime}\right)$.

The composition of ${ }_{x} \lambda$ with the inverse map will be denoted as $\lambda_{x}$; this family defines a right Haar system $\lambda^{-1}$. Any measure $\mu$ on the space of units $\mathcal{T}_{0}$ induces measures $\nu, \nu^{-1}$ on the whole $\mathcal{T}$ through

$$
\int_{\mathcal{T}} f d \nu=\int \lambda(f) d \mu, \quad \int_{\mathcal{T}} f d \nu^{-1}=\int \lambda^{-1}(f) d \mu .
$$

The measure $\mu$ is said to be quasi-invariant if $\nu$ and $\nu^{-1}$ are equivalent measures; in this case the Radon-Nikodym derivative $D_{\mu}=d \nu_{\mu} / d \nu_{\mu}^{-1}$ is called the modular 
function of $\mu$. The function $\log D_{\mu} \in Z^{1}(\mathcal{T}, \mathbb{R})$ turns out to be a groupoid 1cocycle with values in $\mathbb{R}$ and its cohomology class depends only on the equivalence class of $\mu$.

Let $\zeta \in Z^{2}\left(\mathcal{T}, \mathbb{S}^{1}\right)$ be a continuous 2-cocycle. One can define the convolution algebra $C_{c}(\mathcal{T}, \zeta)$ and correspondingly the groupoid $C^{*}$-algebra $C^{*}(\mathcal{T}, \zeta)$. When $\zeta=1$ we denote the convolution and $C^{*}$-algebras with $C_{c}(\mathcal{T})$ and $C^{*}(\mathcal{T})$. Moreover, given a quasi invariant measure $\mu$ one can define the structure of left Hilbert algebra on $L^{2}\left(\mathcal{T}, \nu_{\mu}^{-1}\right)$ such that the modular operator is exactly given by multiplication with $D_{\mu}$.

We are interested in understanding when a given topological groupoid has a (possibly unique) Haar system. We will use the following result.

Definition 2.1. A locally compact groupoid is étale if its unit space is open.

As a consequence, ${ }_{x} \mathcal{T}, \mathcal{T}_{x}$ are discrete for all $x \in \mathcal{T}_{0}$ and if there exists a left Haar system then it is equivalent to the counting measure. We will use in particular the following result (Prop. 2.8 in [22]):

Proposition 2.2. A topological groupoid is étale and admits a left Haar system if and only if $l_{\mathcal{T}}$ is a local homeomorphism.

\section{The quantization framework}

Let $(M, \pi)$ be a Poisson manifold, $\mathcal{G}(M, \pi)$ be its symplectic groupoid, that is assumed to be smooth (this was referred in the previous Section as integrability of $(M, \pi)$; it must not be confused with the notion of integrability of the modular function that we are going to discuss in this Section). Let us fix a volume form $V_{M}$ and let $f_{\chi_{V_{M}}} \in C^{\infty}(\mathcal{G}(M, \pi))$ be the modular function.

We are interested in studying cases where the hamiltonian dynamics of the modular function is integrable, i.e. $f_{\chi_{V_{M}}}$ Poisson commutes with a set $F=$ $\left\{f_{i}\right\}_{i=1}^{\operatorname{dim} \mathcal{G} / 2}$ of hamiltonians $f_{i} \in C^{\infty}(\mathcal{G}(M, \pi))$ in involution. Such functions should be generically non degenerate in the usual sense, i.e. $d f_{1} \wedge \ldots \wedge d f_{n} \neq 0$ on an open and dense subset of $\mathcal{G}(M, \pi)$.

Let us denote with $\mathcal{G}_{F}(M, \pi)$ the topological space of connected contour levels of $F$. We remark that if we denote by $\mathcal{P}_{F}=\left\langle X_{f_{i}}\right\rangle \subset T \mathcal{G}(M, \pi)$ the (generalized) real polarization defined by the hamiltonian vector fields $X_{f_{i}}$ of the $f_{i}$ 's, we have that $\mathcal{G}_{F}(M, \pi)$ is not in general $\mathcal{G}(M, \pi) / \mathcal{P}_{F}$. This is what happens for instance in [2]. We will not necessarily require that $\mathcal{G}_{F}(M, \pi)$ be smooth, we will see later what kind of regularity we need. 
Definition 3.1. The modular function $f_{\chi_{V_{M}}}$ is said to be multiplicatively integrable if there exists on $\mathcal{G}_{F}(M, \pi)$ a topological groupoid structure such that the quotient map $\mathcal{G}(M, \pi) \rightarrow \mathcal{G}_{F}(M, \pi)$ is a groupoid morphism.

Let us recall the prequantization of the symplectic groupoid: this is the ordinary prequantization of the symplectic structure with additional requirements that make it compatible with the groupoid structure. The basic results come from [31, but the presentation is taken from [14]. If $\Lambda$ is a line bundle on $\mathcal{G}$ then with $\partial^{*} \Lambda$ we denote the line bundle $d_{0}^{*} \Lambda \otimes d_{1}^{*} \Lambda^{*} \otimes d_{2}^{*} \Lambda$ on $\mathcal{G}_{2}$.

Definition 3.2. A prequantization of the symplectic groupoid $\mathcal{G}(M, \pi)$ consists of the triple $\left(\Lambda, \nabla ; \zeta_{\Theta_{\mathcal{G}}}\right)$ where $(\Lambda, \nabla)$ is a prequantization of $\mathcal{G}(M, \pi)$ as a symplectic manifold and $\zeta_{\Theta_{\mathcal{G}}}$ is a section of $\partial^{*} \Lambda^{*}$ such that:

i) $\zeta_{\Theta_{\mathcal{G}}}$ has norm one and is multiplicative, i.e. it satisfies the following cocycle condition: for $\left(\gamma_{1}, \gamma_{2}, \gamma_{3}\right) \in \mathcal{G}_{3}(M)$

$$
\begin{aligned}
\partial^{*} \zeta_{\Theta_{\mathcal{G}}}\left(\gamma_{1}, \gamma_{2}, \gamma_{3}\right) & \equiv \zeta_{\Theta_{\mathcal{G}}}\left(\gamma_{1}, \gamma_{2} \gamma_{3}\right) \zeta_{\Theta_{\mathcal{G}}}\left(\gamma_{2}, \gamma_{3}\right) \zeta_{\Theta_{\mathcal{G}}}\left(\gamma_{1}, \gamma_{2}\right)^{-1} \zeta_{\Theta_{\mathcal{G}}}\left(\gamma_{1} \gamma_{2}, \gamma_{3}\right)^{-1} \\
& =1 .
\end{aligned}
$$

ii) $\zeta_{\Theta_{\mathcal{G}}}$ is covariantly constant, i.e. if $\Theta_{\mathcal{G}}$ is a (local) connection form for $\nabla$, then $\zeta_{\Theta_{\mathcal{G}}}$ (locally) satisfies

$$
d \zeta_{\Theta_{\mathcal{G}}}+\left(\partial^{*} \Theta_{\mathcal{G}}\right) \zeta_{\Theta_{\mathcal{G}}}=0 .
$$

We call $\zeta_{\Theta_{\mathcal{G}}}$ the prequantization cocycle defined by $\Theta_{\mathcal{G}}$. From Theorem 3.2 in [31] we get that if $\mathcal{G}(M, \pi)$ is prequantizable as a symplectic manifold and $l_{\mathcal{G}}$-locally trivial, then there exists a unique groupoid prequantization.

The prequantization cocycle can be chosen to be 1 if $\Omega_{\mathcal{G}}$ is multiplicatively exact, i.e. if there exists a primitive $\Theta_{\mathcal{G}}$ such that $\partial^{*} \Theta_{\mathcal{G}}=0$. Theorem 4.2 in [8] shows that $\Omega_{\mathcal{G}}$ is multiplicatively exact if and only if the class of the Poisson bivector $[\pi] \in H_{L P}^{2}(M, \pi)$ is zero.

Finally we say that a leaf $\ell \in \mathcal{G}_{F}(M, \pi)$ satisfies the Bohr-Sommerfeld conditions if the holonomy of $\nabla$ along $\ell$ is trivial. We are able to prove that BohrSommerfeld conditions select a subgroupoid of $\mathcal{G}_{F}(M, \pi)$ only under the following assumption.

Assumption 1: for each couple of composable leaves $\left(\ell_{1}, \ell_{2}\right) \in \mathcal{G}_{F}(M, \pi)_{2}$ the map $m_{\mathcal{G}}: \ell_{1} \times \ell_{2} \cap \mathcal{G}(M, \pi)_{2} \rightarrow \ell_{1} \ell_{2}$ induces a surjective map in homology.

This assumption is verified by the integrable model we are going to consider in the following Sections.

Lemma 3.3. If Assumption 1 is satisfied then the set of Bohr-Sommerfeld leaves $\mathcal{G}_{F}^{b s}(M, \pi) \subset \mathcal{G}_{F}(M, \pi)$ inherits the structure of a topological groupoid. 
Proof. Let $\gamma$ be a loop in the leaf $\ell=\ell_{1} \ell_{2}$ and let $\gamma(t)=\gamma_{1}(t) \gamma_{2}(t)$, for $\left(\gamma_{1}(t), \gamma_{2}(t)\right) \in \ell_{1} \times \ell_{2} \cap \mathcal{G}(M, \pi)_{2}$. Then we have that

$$
\int_{\gamma} \Theta_{\mathcal{G}}=\int_{0}^{1} d t\left\langle\Theta_{\mathcal{G}}, m_{*}\left(\dot{\gamma}_{1} \oplus \dot{\gamma}_{2}\right)\right\rangle=\int_{\left(\gamma_{1}, \gamma_{2}\right)} \partial^{*} \Theta_{\mathcal{G}}+\int_{\gamma_{1}} \Theta+\int_{\gamma_{2}} \Theta
$$

Since $\partial^{*} \Theta_{\Theta_{\mathcal{G}}}$ satisfies (41) then we have that if $\ell_{1}, \ell_{2} \in \mathcal{G}_{F}^{b s}(M, \pi)$ then $\ell_{1} \ell_{2} \in$ $\mathcal{G}_{F}^{b s}(M, \pi)$.

We refer to $\mathcal{G}_{F}^{b s}(M, \pi)$ as the groupoid of Bohr-Sommerfeld leaves.

Assumption 2: the groupoid of Bohr-Sommerfeld leaves $\mathcal{G}_{F}^{b s}(M, \pi)$ admits a Haar system.

If Assumption 1 and 2 are satisfied then we get that the groupoid of BohrSommerfeld leaves $\mathcal{G}_{F}^{b s}(M, \pi)$ admits a Haar system and inherits the modular cocycle $f_{\chi_{V_{M}}}$. Moreover, if the prequantization cocycle $\zeta_{\Theta_{\mathcal{G}}}$ can be chosen to be $F$-invariant it descends to $\mathcal{G}_{F}^{b s}$ too. These data give a quantization of the Poisson structure. Indeed, by applying the results of Renault's theory summarized in the previous Section, we can define the convolution algebra $C_{c}\left(\mathcal{G}_{F}^{b s}, \zeta_{\Theta_{G}}\right)$. Moreover, we can determine the quasi invariant measure $\mu_{V_{M}}$ whose modular cocycle is $f_{\chi_{V_{M}}}$ and then define the left Hilbert algebra on $L^{2}\left(\mathcal{G}_{F}^{b s}, \nu_{\mu_{V_{M}}}^{-1}\right)$.

In the rest of the paper, we will discuss a concrete example where such a quantization exists.

\section{A family of $S U(n+1)$ covariant Poisson struc- tures on $\mathbb{C} P_{n}$}

We introduce in this Section the basic example that we are going to quantize. It is a family $\pi_{t}, t \in[0,1]$, of Poisson structures on the complex projective space $\mathbb{C} P_{n}$ that are embeddable homogeneous spaces of the standard Poisson structure on $S U(n+1)$.

We will first recall the standard Poisson structure on $S U(n+1)$ (see [19]) and then introduce a family of coisotropic subgroups $U_{t}(n) \subset S U(n+1)$.

The standard multiplicative Poisson tensor of $S U(n+1)$ can be described intrinsically integrating a $\wedge^{2} \mathfrak{s u}$ valued Lie-algebra 2 -cocycle; here, in view of later applications, we describe it in terms of the dressing transformation of $S B(n+$ $1, \mathbb{C})$ on $S U(n+1)$. Let us start with the Iwasawa decomposition

$$
S L(n+1, \mathbb{C})=S U(n+1) \times S B(n+1, \mathbb{C}), \quad S B(n+1, \mathbb{C})=A_{n+1} N_{n+1},
$$

where $A_{n+1}=\left\{\operatorname{diag}\left(v_{1}, \ldots, v_{n+1}\right), v_{i}>0, v_{1} v_{2} \ldots v_{n+1}=1\right\}$ and $N_{n+1}$ is the group of upper diagonal complex matrices with 1 on the diagonal. It is easily shown that $\operatorname{pr}_{A_{n+1}}: S B(n+1, \mathbb{C}) \rightarrow A_{n+1}$ is a group morphism. 
The Lie algebras $\mathfrak{s u}(n+1)$ and $\mathfrak{s} \mathfrak{b}(n+1)$ are dual to each other through the pairing $\langle X, \xi\rangle=\operatorname{Im} \operatorname{Tr}(X \xi)$, for $X \in \mathfrak{s u}(n+1), \xi \in \mathfrak{s} \mathfrak{b}(n+1, \mathbb{C})$. We will therefore often identify implicitly $\mathfrak{s b}$ with $\mathfrak{s u}^{*}$. We will denote by $p_{1,2}$ the projections from $\mathfrak{s l}$ to $\mathfrak{s u}$ and $\mathfrak{s b}$ respectively. As said above, any $d \in S L(n+1, \mathbb{C})$ can be Iwasawa decomposed into $g \gamma$ with $g \in S U(n+1)$ and $\gamma \in S B(n+1, \mathbb{C})$. By taking the product $\gamma \cdot g$ and rewriting it using the Iwasawa decomposition as $\gamma \cdot g={ }^{\gamma} g \cdot \gamma^{g}$, with ${ }^{\gamma} g \in S U$ and $\gamma^{g} \in S B$, one has defined the dressing transformation

$$
S B(n+1, \mathbb{C}) \times S U(n+1)) \rightarrow S U(n+1) ; \quad(\gamma, g) \rightarrow{ }^{\gamma} g .
$$

If $\xi \in \mathfrak{s} \mathfrak{b}$ then one has $\xi g=g A d_{g^{-1}} \xi$, thus the infinitesimal dressing transformation generated by $\xi$ is

$$
l_{g}\left(p_{1} A d_{g^{-1}} \xi\right)
$$

where $g \in S U(n+1)$ and $l_{g}$ denotes left multiplication by $g$. Now regard $\xi$ as in $\mathfrak{s u}^{*}$, one has $A d_{g}^{*} \xi=p_{2} A d_{g} \xi$, which can also be seen by using the pairing. The Poisson tensor, when evaluated on two right invariant 1-forms $\left(r_{g^{-1}}\right)^{*} \xi_{1,2}$, is given by the paring of the 1 -form $\left(A d_{g}\right)^{*} \xi_{2}$ with the vector field (5) generated by $\xi_{1}$, or in formulae:

$$
\pi\left(\left(r_{g^{-1}}\right)^{*} \xi_{1},\left(r_{g^{-1}}\right)^{*} \xi_{2}\right)=-\left\langle p_{1} A d_{g^{-1}} \xi_{1}, p_{2} A d_{g^{-1}} \xi_{2}\right\rangle .
$$

From this formula one can show directly that $\pi$ is Poisson and it is furthermore multiplicative $\pi(g h)=\left(l_{g}\right)_{*} \pi(h)+\left(r_{h}\right)_{*} \pi(g)$.

Dually, changing the roles of the two groups, one defines the dressing transformation of $S U(n+1)$ on $S B(n+1, \mathbb{C})$, and can write down a multiplicative Poisson tensor for the latter. In this way $S B(n+1, \mathbb{C})$ becomes the dual simply connected Poisson-Lie group of $S U(n+1)$.

Let us denote for $t \in[0,1]$

$$
\sigma_{t}=\left(\begin{array}{ccc}
\sqrt{1-t} & 0 & \sqrt{t} \\
0 & \operatorname{id}_{n-1} & 0 \\
-\sqrt{t} & 0 & \sqrt{1-t}
\end{array}\right) \in S U(n+1) .
$$

Using the formula for the Poisson tensor (6), it can be shown that the subgroups

$$
U_{t}(n) \equiv \sigma_{t} S(U(1) \times U(n)) \sigma_{t}^{-1} \subset S U(n+1)
$$

are coisotropic for all $t \in[0,1]$ (this was first proved in [27] at the infinitesimal level). Let us denote with $\mathfrak{u}_{t}(n)=$ Lie $U_{t}(n)$. Since $U_{t}(n)$ is coisotropic, $\mathfrak{u}_{t}(n)^{\perp}$ is a Lie subalgebra and we will denote with $U_{t}(n)^{\perp} \subset S B(n+1, \mathbb{C})$ the subgroup integrating $\mathfrak{u}_{t}(n)^{\perp} \subset \mathfrak{s} \mathfrak{b}(n+1, \mathbb{C})$. An explicit expression for $\mathfrak{u}_{t}(n)^{\perp}$ can be deduced from [7], page 16. As a consequence of coisotropy there is a uniquely 
defined Poisson structure $\pi_{t}$ on $\mathbb{C} P_{n}=U_{t}(n) \backslash S U(n+1)$ such that the quotient $\operatorname{map} p_{t}: S U(n+1) \rightarrow \mathbb{C} P_{n}$ is Poisson.

It has to be remarked that Poisson structures corresponding to different values of $t$ are not totally unrelated. Let $X_{j}, j=1 \ldots n+1$ denote the homogeneous coordinates of $\mathbb{C} P_{n}$.

Lemma 4.1. The diffeomorphism $\psi: \mathbb{C} P_{n} \rightarrow \mathbb{C} P_{n}$ defined as

$$
\psi\left[X_{1}, \ldots, X_{n+1}\right]=\left[X_{n+1}, \ldots, X_{1}\right]
$$

sends $\pi_{t}$ to $-\pi_{1-t}$.

Proof. Let, in fact, $J=\left(i \delta_{i, n+1-i}\right) \in S U(n+1)$ be the matrix with all antidiagonal elements equal to $i$. We have clearly that $\psi$ is just the right multiplication by $J^{-1}$. Then a direct computation shows that $p_{t}\left(J g J^{-1}\right)=\psi\left(p_{1-t}(g)\right)$ for all $g \in S U(n+1)$. On the other hand one has that $A \mapsto J A J^{-1}$ is an anti-Poisson map, which therefore induces an anti-Poisson map on complex projective spaces.

In general $\pi_{t}$ and $\pi_{t^{\prime}}$ for $t^{\prime} \neq 1-t$ are not Poisson or anti Poisson diffeomorphic. This is shown, for instance, for $\mathbb{C} P_{1}$ in [4].

The family of covariant Poisson structures $\pi_{t}$ on $\mathbb{C} P_{n}$ exhibits a sharply different behaviour, depending on whether $t$ is one of the limiting values $t=0,1$, which will be called the standard or Bruhat-Poisson structure, or $t \in(0,1)$, that will be referred to as the non standard case. This is best described by analyzing the corresponding symplectic foliation. It is an interesting fact that such foliation is essentially determined by the images under the projection of the Poisson subgroups of $S U(n+1)$. For each $k=1, \ldots, n$, let

$$
G_{k}=S(U(k) \times U(n+1-k)) \subset S U(n+1) ; \quad P_{k}(t)=p_{t}\left(G_{k}\right) \subset \mathbb{C} P_{n} .
$$

Each $G_{k}$ is a Poisson subgroup so that $P_{k}(t)$ is a Poisson submanifold of $\mathbb{C} P_{n}$. As can be easily deduced from [29], Proposition 2.1, this exhausts the list of maximal (with respect to inclusion) Poisson subgroups of $S U(n+1)$. We will explicitly describe the Poisson submanifolds $P_{k}(t)$.

When $t=0$, i.e. in the case of the Bruhat-Poisson structure, Poisson submanifolds $P_{k}(0) \sim \mathbb{C} P_{k-1}$ are contained one inside the other giving rise to the following chain of Poisson embeddings:

$$
\{\infty\}=\mathbb{C} P_{0} \subset \mathbb{C} P_{1} \subset \ldots \mathbb{C} P_{n}
$$

where $\mathbb{C} P_{k}$ corresponds to $X_{j}=0$ for $j>k$ so that $\infty=[1,0, \ldots, 0]$. The symplectic foliation, then, corresponds to the Bruhat decomposition

$$
\mathbb{C} P_{n}=\bigcup_{i=1}^{n+1} \mathcal{S}_{i},
$$


where each $\mathcal{S}_{i}$ can be described as $\mathcal{S}_{i}=\left\{\left[X_{1}, \ldots, X_{i}, 0, \ldots 0\right], X_{i} \neq 0\right\} \subset \mathbb{C} P_{i-1}$. Thus we have one contractible symplectic leaf in each even dimension, which turns out to be symplectomorphic to standard $\mathbb{C}^{i}$. The maximal symplectic leaf (the maximal cell) $\mathcal{S}_{n+1}$ is open and dense in $\mathbb{C} P_{n}$. On such $2 n$-dimensional open cell $\mathcal{S}_{n+1}$ it is possible to define the so-called Lu's coordinates $\left\{y_{i}\right\}_{i=1}^{n}$ as follows (see Appendix $\mathrm{A}$ for more details). Let $z_{i}=X_{i} / X_{n+1}, 1 \leq i \leq n$ be the standard affine coordinates on this cell and let us define $y_{n}=z_{n}$ and for $i<n$

$$
y_{i}=\frac{z_{i}}{\sqrt{1+\sum_{i<j \leq n}\left|z_{j}\right|^{2}}} .
$$

In terms of such coordinates the Bruhat Poisson structure restricted to $\mathcal{S}_{n+1}$ reads

$$
\left.\pi_{0}\right|_{\mathcal{S}_{n+1}}=i \sum_{i=1}^{n}\left(1+\left|y_{i}\right|^{2}\right) \partial_{y_{i}} \wedge \partial_{\bar{y}_{i}} .
$$

Let us now move to the non standard case. Most of this description is contained in [7, to which we refer for unproven statements. By direct computation we have that if $g \in G_{k}$ then $p_{t}(g)=\left[X_{1}, \ldots, X_{n+1}\right]=\left[(1,0, \ldots, 0) \sigma_{t}^{-1} g\right] \in P_{k}(t)$, satisfies

$$
F_{k, t}\left(X_{1}, \ldots X_{n+1}\right)=t \sum_{i=1}^{k}\left|X_{i}\right|^{2}-(1-t) \sum_{i=k+1}^{n+1}\left|X_{i}\right|^{2}=0
$$

Since $F_{k+1, t}-F_{k, t}=\left|X_{k+1}\right|^{2}$ we have

$$
F_{n, t} \geq F_{n-1, t} \geq \ldots \geq F_{1, t} .
$$

Let $P_{\text {sing }}=\bigcup_{k=1}^{n} P_{k}(t)$, we can easily see that $\mathbb{C} P_{n} \backslash P_{\text {sing }}$ is a disconnected union of $(n+1)$ affine spaces. Each of the $P_{k}(t)$ is a union of lower-dimensional symplectic leaves; we recall the following facts from [7]:

1. $P_{1}(t)$ and $P_{n}(t)$ are Poisson diffeomorphic to the standard Poisson sphere $\mathbb{S}^{2 n-1}$, by which we mean the Poisson spheres first described in [30], having an $\mathbb{S}^{1}$ family of symplectic leaves in each even dimension.

2. if $1<k<n$ then $P_{k}(t)=\mathbb{S}^{2 k-1} \times \mathbb{S}^{2(n-k)+1} / \mathbb{S}^{1}$ where the two odddimensional spheres are endowed with the standard odd Poisson sphere structure and $\mathbb{S}^{1}$ acts diagonally.

It is relevant for what follows to remark here that as Poisson manifolds $P_{k}(t)$ do not depend on $t$ (up to Poisson diffeomorphism); the dependence on $t$ is manifested in the embeddings. What was not detailed in [7] is the way in which 
such Poisson submanifolds intersect along lower dimensional symplectic leaves. Let us remark the following. First, if $k<h$, then $P_{k}(t) \cap P_{h}(t)=\bigcap_{i=k}^{h} P_{i}(t)$ and this is a simple consequence of inequalities (13). Furthermore, from the analysis of the symplectic foliation of odd-dimensional Poisson spheres one can prove that the singular part of the symplectic foliation of each $P_{k}(t)$ is just $P_{k}(t) \cap P_{k-1}(t) \cup P_{k}(t) \cap P_{k+1}(t)$; the limiting cases $k=1, n$ of course behave differently in that only one of the two terms in the union appears. This can be summarized by saying that the foliation resembles more a stratification where the strata of symplectic leaves of dimension $2 m$ are given by the intersections of $n-m$ consecutive Poisson submanifolds $P_{k}(t)$; furthermore each $\bigcap_{i=k}^{k+n-m} P_{i}(t)$ is equal to the intersection of $P_{k}(t)$ with the linear subspace $X_{k+1}=\ldots=X_{k+n-m-1}=0$. Thus, for example, the subset of 0 -dimensional symplectic leaves equals

$$
\bigcap_{i=1}^{n} P_{i}(t)=\left\{\left.\left[X_{1}, 0, \ldots, 0, X_{n+1}\right]|t| X_{1}\right|^{2}-(1-t)\left|X_{n+1}\right|^{2}=0\right\} \simeq \mathbb{S}^{1}
$$

In each even dimension lower than $2 n$ there appears continuous families of symplectic leaves, in sharp contrast with the standard case.

In low dimensions we can say that non standard $\mathbb{C} P_{1}$ contains a singular $\mathbb{S}^{1}$ of 0-dimensional leaves of equation $t\left|X_{1}\right|^{2}-(1-t)\left|X_{2}\right|^{2}=0$, separating two 2dimensional symplectic cells. As for non standard $\mathbb{C} P_{2}$, it contains two copies of $\mathbb{S}^{3}$ separating three affine 4-dimensional cells: the two embedded Poisson spheres $\mathbb{S}^{3}$ are made of two distinct $\mathbb{S}^{1}$-families of 2 -dimensional symplectic leaves and are intersecting along a common circle $\mathbb{S}^{1}$ of 0 -dimensional leaves. The singular part of the symplectic foliation for $\mathbb{C} P_{3}$ is summarized in the following diagram, which is, of course, also the starting diagram of leaves of dimension up to 4 in each higher dimensional $\mathbb{C} P_{n}$ (and can be easily extended in any dimension):

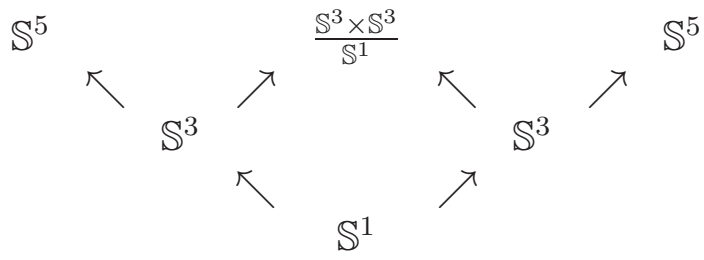

We summarize the description of the symplectic foliation of $\left(\mathbb{C} P_{n}, \pi_{t}\right)$ in the following Proposition.

Proposition 4.1. For $t \in(0,1),\left(\mathbb{C} P_{n}, \pi_{t}\right)$ decomposes in the following regular Poisson submanifolds

$$
\mathbb{C} P_{n}=\bigcup_{r, s \geq 0, r+s=0}^{n} \mathbb{C} P_{n}^{(r, s)}
$$


where for $r+s=n, \mathbb{C} P_{n}^{(r, s)}$ is an open connected component of $\mathbb{C} P_{n} \backslash P_{\text {sing }}$, and for $r+s<n$

$$
\mathbb{C} P_{n}^{(r, s)}=\cap_{j=1}^{n-r-s} P_{r+j}(t) \backslash P_{r}(t) \cup P_{n+1-s}(t) \quad .
$$

For $r+s=n,\left(\mathbb{C} P_{n}^{(r, s)}, \pi_{t}\right)$ is non degenerate. For $r+s<n,\left(\mathbb{C} P_{n}^{(r, s)}, \pi_{t}\right)$ is Poisson diffeomorphic to $\mathcal{S}_{r, s}^{2(r+s)} \times \mathbb{S}^{1}$, where $\mathcal{S}_{r, s}^{2(r+s)}$ is a symplectic manifold of dimension $2(r+s)$.

We will need the following fact.

Proposition 4.2. All symplectic leaves of $\left(\mathbb{C} P_{n}, \pi_{t}\right)$, for $t=[0,1]$, are contractible.

Proof. For $t=0,1$ the symplectic foliation corresponds to the Bruhat decomposition so that the result is straightforward. Let us consider $t \in(0,1)$. Let us discuss first the open symplectic leaves $\mathbb{C} P_{n}^{(r, n-r)}$ of dimension $2 n$. For $r=0, n$ they are defined by $0<F_{1, t}$ and $F_{n, t}<0$ respectively; it is easy to check that they are open disks. For $0<r<n, \mathbb{C} P_{n}^{(r, n-r)}$ is characterized by the inequality $F_{r, t}<0<F_{r+1, t}$, that implies $F_{r+1, t}-F_{r, t}=\left|X_{r+1}\right|^{2} \neq 0$. So let us define for $\lambda \in[0,1]$

$$
\epsilon\left(\lambda,\left[X_{1}, \ldots X_{n+1}\right]\right)=\left[\lambda X_{1}, \ldots, \lambda X_{r}, X_{r+1}, \lambda X_{r+2}, \ldots, \lambda X_{n+1}\right] \quad .
$$

It is straightforward to verify that $\epsilon$ preserves the inequalities, i.e. $\epsilon:[0,1] \times$ $\mathbb{C} P_{n}^{(r, n-r)} \rightarrow \mathbb{C} P_{n}^{(r, n-r)}, \epsilon(1, p)=p$ and $\epsilon(0, p)=[0, \ldots, 0,1,0, \ldots, 0]$ for all $p \in$ $\mathbb{C} P_{n}^{(r, n-r)}$.

Let us consider now the singular part. $\mathbb{C} P_{n}^{(r, s)}$ is identified by

$$
F_{r, t}<0=F_{r+1, t}=F_{r+2, t}=\ldots=F_{n-s, t}<F_{n-s+1, t}
$$

By using the definition of the $F$ 's it easy to conclude that $\left[X_{1}, \ldots, X_{n+1}\right] \in \mathbb{C} P_{n}^{(r, s)}$ iff $X_{r+2}=X_{r+3}=\ldots=X_{n-s}=0, X_{r+1}, X_{n-s+1} \neq 0$ and

$$
t \sum_{j=1}^{r+1}\left|X_{j}\right|^{2}=(1-t) \sum_{j=n-s+1}^{n+1}\left|X_{j}\right|^{2}
$$

Note if $r+s=n-1$ no homogeneous coordinate $X$ is zero, but it is easy to adjust the following construction also to this case. We can then describe $\mathbb{C} P_{n}^{(r, s)}=\cup_{\mu \in \mathbb{S}^{1}} \mathcal{S}_{r, s}^{2(r+s)}(\mu)$ where

$$
\mathcal{S}_{r, s}^{2(r+s)}(\mu)=\left\{(z, \tilde{z})=\left[z_{1}, \ldots, z_{r}, z_{r+1}=\rho \mu, 0, \ldots, 0,1, \tilde{z}_{n-s+2}, \ldots, \tilde{z}_{n+1}\right]\right.
$$




$$
\left.\rho>0, \quad t \sum_{j=1}^{r}\left|z_{j}\right|^{2}+t \rho^{2}=(1-t)\left(1+\sum_{j=n-s+2}^{n+1}\left|\tilde{z}_{j}\right|^{2}\right)\right\}
$$

is a symplectic leaf parametrized by $\mu \in \mathbb{S}^{1}$. From the properties of the action of the Cartan subgroup, we conclude that $z_{r+1} \rightarrow z_{r+1} e^{i \phi}$ is a Poisson diffeomorphism from $\mathcal{S}_{r, s}^{2(r+s)}(\mu)$ to $\mathcal{S}_{r, s}^{2(r+s)}\left(\mu e^{i \phi}\right)$. This proves that $\mathbb{C} P_{n}^{(r, s)}=\mathcal{S}_{r, s}^{2(r+s)}(1) \times \mathbb{S}^{1}$ as a Poisson manifold.

The following map $\epsilon:[0,1] \times \mathcal{S}_{r, s}^{2(r+s)}(\mu) \rightarrow \mathcal{S}_{r, s}^{2(r+s)}(\mu)$

$$
\epsilon(\lambda,(z, \tilde{z}))=\left(\epsilon_{0}(z, \lambda) z, \lambda \tilde{z}\right) \quad \epsilon_{0}(z, \lambda)=\sqrt{\lambda^{2}+\frac{(1-t)\left(1-\lambda^{2}\right)}{t \sum_{i}^{r}\left|z_{i}\right|^{2}+t \rho^{2}}}
$$

contracts $\mathcal{S}_{r, s}^{2(r+s)}(\mu)$ to the open disk $\left\{\left(z_{1}, \ldots, z_{r}\right), \quad \sum_{i=1}^{r}\left|z_{i}\right|^{2}<(1-t) / t\right\}$.

Finally let us describe the (ssc) symplectic groupoid integrating $\left(\mathbb{C} P_{n}, \pi_{t}\right)$. It can be constructed as a symplectic reduction of the Lu-Weinstein symplectic groupoid structure on $S L(n+1, \mathbb{C})$ integrating the Poisson Lie group $(S U(n+$ $1), \pi$ ), along the lines described in Subsection 2.2. So that we have

$$
\mathcal{G}\left(\mathbb{C} P_{n}, \pi_{t}\right)=\left\{[g \gamma], g \in S U(n+1), \gamma \in S B(n+1, \mathbb{C}),{ }^{g} \gamma \in U_{t}(n)^{\perp}\right\} \quad,
$$

where we denoted with $[g \gamma]$ the class of $g \gamma \in S L(n+1, \mathbb{C})$ under the quotient map $S L(n+1, \mathbb{C}) \rightarrow U_{t}(n) \backslash S L(n+1, \mathbb{C})$. As a smooth manifold, it can be described as the fibre bundle over $\mathbb{C} P_{n}$ with fibre $U_{t}(n)^{\perp}$ associated to the homogeneous principal bundle with the dressing action of $U_{t}(n)$ on $U_{t}(n)^{\perp}$. We will denote the symplectic form as $\Omega_{\mathcal{G}}$. Since the $l$ (or $r$ ) fibers are diffeomorphic to $U_{t}(n)^{\perp}$ that is contractible, the restriction of the symplectic form $\Omega_{\mathcal{G}}$ to the $l$-fibres is exact; by applying Corollary 5.3 of [8] we conclude that $\Omega_{\mathcal{G}}$ is exact.

The homogenous action of $S U(n+1)$ on $\mathbb{C} P_{n}$ is Poisson; thus it can be lifted to an action on the symplectic groupoid given by right $S U(n+1)$ multiplication. This action is symplectic and admits Lu momentum map. We are interested in its restriction to the action of the Cartan subgroup $\mathbb{T}^{n}$, which is hamiltonian in the usual sense. Under the identification $\mathfrak{t}_{n}^{*}=\operatorname{Lie} A_{n+1}$, the momentum map $h: \mathcal{G}\left(\mathbb{C} P_{n}, \pi_{t}\right) \rightarrow \mathfrak{t}_{n}^{*}$ is defined for each $[g \gamma] \in \mathcal{G}\left(\mathbb{C} P_{n}, \pi_{t}\right)$ as

$$
h[g \gamma]=\log \operatorname{pr}_{A_{n+1}}(\gamma) .
$$

For each $H \in \mathfrak{t}_{n}$ we denote the component of $h$ along $H$ as $h_{H}[g \gamma]=\langle H, h[g \gamma]\rangle=$ $\operatorname{Im} \operatorname{Tr}\left(H \log \operatorname{pr}_{A_{n+1}} \gamma\right)$. It is easy to check that $h_{H}$ is the $\mathbb{R}$-valued groupoid cocycle that lifts the Poisson fundamental vector field realizing the $\mathfrak{t}_{n}$ action on $\mathbb{C} P_{n}$. 


\section{The bihamiltonian system on $\mathbb{C} P_{n}$}

We introduce here the integrable model relevant for the quantization of the covariant Poisson structures introduced in the previous section. It is, basically, the toric structure on $\mathbb{C} P_{n}$ defined by the Cartan subgroup action; we feel however necessary to emphasize its bihamiltonian structure.

Let $\pi_{\lambda}$ be the inverse of the Fubini-Study symplectic form $\omega_{\lambda}$ on $\mathbb{C} P_{n}$ defined in (35). We know from the results of [16], valid on compact Hermitian symmetric spaces, that the Bruhat-Poisson structure $\pi_{0}$ and $\pi_{\lambda}$ are compatible, i.e.

$$
\left[\pi_{0}, \pi_{\lambda}\right]=0
$$

so that a Poisson pencil $\pi_{0}+t \pi_{\lambda} t \in \mathbb{R}$, of $S U(n+1)$-covariant Poisson structures is defined (see also [13] for an alternative proof). We are going to show that the family of Poisson structures $\pi_{t}$ that we discussed in Section 4 coincide with this Poisson pencil for $t \in[0,1]$.

Lemma 5.1. For each $t \in[0,1], \pi_{t}=\pi_{0}+t \pi_{\lambda}$.

Proof. The proof is quite general and depends on the fact that there exists only one Poisson pencil of $S U(n+1)$-covariant Poisson strcutures on $\mathbb{C} P_{n}$. In fact given the two covariant Poisson structures $\pi_{t}$ and $\pi_{0}$ then their difference is a $S U(n+1)$-invariant 2-tensor. In principle there is no guarantee that such tensor is Poisson; however, as proven in [27], up to scalar multiples, the only $S U(n+1)$ invariant 2-tensor on $\mathbb{C} P_{n}$ is the inverse of the Fubini-Study symplectic form.

In Appendix B we give a self contained alternative proof of the above Lemma based on a direct computation; this will fix the scalar multiplying $\pi_{\lambda}$ appearing in the above proof.

From now on, we will use the notation $\pi_{t}=\pi_{0}+t \pi_{\lambda}$ for $t \in \mathbb{R}$, keeping in mind that $\pi_{t}$ is obtained as a quotient by a coisotropic subgroup only for $t \in[0,1]$.

From the general theory of bihamiltonian systems (see [20] for an introduction) we get that the recursion operator $J_{\lambda}=\pi_{0} \circ \omega_{\lambda}$ has vanishing Nijenhuis torsion, i.e. for each couple $\left(v_{1}, v_{2}\right)$ of vector fields

$$
T\left(J_{\lambda}\right)\left(v_{1}, v_{2}\right)=\left[J_{\lambda} v_{1}, J_{\lambda} v_{2}\right]-J_{\lambda}\left(\left[J_{\lambda} v_{1}, v_{2}\right)+\left[v_{1}, J_{\lambda} v_{2}\right]-J_{\lambda}\left[v_{1}, v_{2}\right]\right)=0 .
$$

Let us define $I_{k}=\frac{1}{k} \operatorname{Tr} J_{\lambda}^{k}, k=1 \ldots n$. It is shown in Section 4 of [20] that these global functions satisfy the so called Lenard recursion relations

$$
d I_{k+1}=J_{\lambda}^{*} d I_{k}
$$

moreover, they are in involution with respect to both Poisson structures $\pi_{0}$ and $\pi_{\lambda}$. Since the modular vector field is the divergence of the Poisson tensor, we can 
conclude that the modular vector field with respect to the Fubini-Study volume form $V_{F S}=\frac{1}{n !} \omega_{\lambda}^{n}$ is independent on $t$. Moreover, it is a consequence of Theorem 3.5 of [11] that this modular vector field is the $\pi_{\lambda}$ hamiltonian vector field of $I_{1}$, i.e.

$$
\chi_{F S} \equiv \operatorname{div}_{V_{F S}} \pi_{t}=\pi_{\lambda}\left(d \operatorname{Tr} I_{1}\right) .
$$

We are going to show that the fundamental vector fields of the Cartan action solve the spectral problem for the recursion operator.

The homogeneous action of the Cartan subgroup $\mathbb{T}^{n} \subset S U(n+1)$ is hamiltonian with respect to $\pi_{\lambda}$ with hamiltonian $c: \mathbb{C} P_{n} \rightarrow \mathfrak{t}_{n}^{*}$. The component of $c$ along $H \in \mathfrak{t}_{n}$ reads

$$
c_{H}[g] \equiv\langle c[g], H\rangle=\operatorname{Tr}\left(g^{-1} \lambda g H\right),
$$

where $\lambda$ is defined in (36). Let us introduce the following basis $\left\langle H_{k}, k=1, \ldots n\right\rangle$ for $\mathfrak{t}_{n}=\operatorname{Lie}^{n}$, where for each $k$ we have

$$
H_{k}=\frac{i}{n+1}\left(\begin{array}{cc}
(n+1-k) \mathrm{id}_{k} & 0 \\
0 & -k \mathrm{id}_{n+1-k}
\end{array}\right) .
$$

In terms of homogeneous and Lu's coordinates we have

$$
c_{k} \equiv c_{H_{k}}+\frac{k}{n+1}=\frac{\sum_{1 \leq j \leq k}\left|X_{j}\right|^{2}}{\sum_{j}\left|X_{j}\right|^{2}}=1-\Pi_{i=1}^{k} \frac{1}{1+\left|y_{i}\right|^{2}},
$$

respectively. It is clear that

$$
0 \leq c_{1} \leq c_{2} \ldots \leq c_{n} \leq 1
$$

so that the image of the momentum map is the standard simplex $\Delta_{n} \subset \mathbb{R}^{n}$.

The action of the Cartan is hamiltonian with respect to $\pi_{\lambda}$ but only Poisson with respect to $\pi_{t}$ for all $t \in[0,1]$, i.e. the fundamental vector fields of the action define non trivial classes in the Lichnerowicz-Poisson cohomology. It is possible to define local hamiltonians, as we are going to show in the following Proposition.

Proposition 5.1. On each open subset of $\mathbb{C} P_{n}$ where $c_{k} \neq 1-t$, the function

$$
b_{k}=\log \left|c_{k}-1+t\right|
$$

is a local hamiltonian for the fundamental vector field of $H_{k}$, i.e. $\sigma_{H_{k}}=\left\{b_{k},-\right\}_{\pi_{t}}$. Moreover,

$$
J_{\lambda}\left(\sigma_{H_{k}}\right)=\left(c_{k}-1\right) \sigma_{H_{k}}
$$


Proof. Let $f \in C^{\infty}\left(\mathbb{C} P_{n}\right)$; then we compute

$$
\left\{b_{k}, f\right\}_{\pi_{t}}=\frac{\partial b_{k}}{\partial c_{k}}\left\{c_{k}, f\right\}_{\pi_{t}}=\frac{\partial b_{k}}{\partial c_{k}}\left(\left\{c_{k}, f\right\}_{\pi_{0}}+t \sigma_{H_{k}}(f)\right) .
$$

If we assume also that $X_{n+1} \neq 0$, we can use Lu coordinates $\left\{y_{i}\right\}$, defined in (10), where the Poisson bivector $\pi_{0}$ takes the form (11). This assumption does not spoil the global validity of the result and the calculation is done in this way because the computation in these coordinates is particularly transparent, while a coordinate free computation is lengthy and unilluminating.

Let us first remark that in these coordinates

$$
\sigma_{H_{k}}=i \sum_{j=1}^{k}\left(y_{j} \partial_{y_{j}}-\bar{y}_{j} \partial_{\bar{y}_{j}}\right)
$$

We then compute

$$
\left\{b_{k}, f\right\}_{\pi_{t}}=\frac{1}{c_{k}-1+t}\left(-\frac{1}{\Pi_{j=1}^{k}\left(1+\left|y_{j}\right|^{2}\right)}+t\right) \sigma_{H_{k}}(f)=\sigma_{H_{k}}(f)
$$

where in the last passage we used (20). Finally, if we denote $b_{k}^{0}=\left.b_{k}\right|_{t=0}=$ $\log \left|c_{k}-1\right|$ then we compute

$$
J_{\lambda}\left(\sigma_{H_{k}}\right)=\pi_{0} d c_{k}=\frac{\partial c_{k}}{\partial b_{k}^{0}} \pi_{0} d b_{k}^{0}=\frac{\partial c_{k}}{\partial b_{k}^{0}} \sigma_{H_{k}}=\left(c_{k}-1\right) \sigma_{H_{k}},
$$

where in the first equality we used the definition of the recursion operator $J_{\lambda}=$ $\pi_{0} \circ \omega_{\lambda}$.

We then conclude the following result on the modular vector field and class of $\left(\mathbb{C} P_{n}, \pi_{t}\right)$.

Lemma 5.2. The modular vector field of $\left(\mathbb{C} P_{n}, \pi_{t}\right), t \in \mathbb{R}$, with respect to the Fubini-Study volume form is

$$
\chi_{F S}=\sum_{i=1}^{n} \sigma_{H_{i}}
$$

where $\sigma_{H_{i}}$ is the fundamental vector field of $H_{i} \in \mathfrak{t}_{n}$. The modular class is non zero for each $t \in[0,1]$.

We note that $\sum_{i=1}^{n} H_{i}=\frac{1}{2} \sum_{\alpha>0} \alpha$, the half sum of positive roots. 


\section{The quantization of $\left(\mathbb{C} P_{n}, \pi_{t}\right)$}

We introduce here the multiplicative integrable model on the symplectic groupoid $\mathcal{G}\left(\mathbb{C} P_{n}, \pi_{t}\right)$, where $\pi_{t}=\pi_{0}+t \pi_{\lambda}$ for $t \in[0,1]$; we compute its groupoid of BohrSommerfeld leaves and prove that it gives the quantization of $\left(\mathbb{C} P_{n}, \pi_{t}\right)$.

\subsection{The multiplicative integrable model}

Let us introduce the integrable system. The action of the Cartan $\mathbb{T}^{n} \subset S U(n+1)$ on $\left(\mathbb{C} P_{n}, \pi_{\lambda}\right)$ is hamiltonian with momentum map $c$ defined in (20); similarly the right action on $\mathcal{G}\left(\mathbb{C} P_{n}, \pi_{t}\right)$ is hamiltonian with momentum map $h$ given in (18). With $\left\{c_{i}\right\}$ and $\left\{h_{i}\right\}$ we denote the components of the momentum maps along the basis $\left\langle H_{i}\right\rangle$ defined in (19). We remark that since $\operatorname{pr}_{A_{n+1}}: S B(n+1, \mathbb{C})=$ $A_{n+1} N_{n+1} \rightarrow A_{n+1}$ is a group homomorphism the components of $h$ are groupoid cocycles. In particular, as a consequence of Lemma 5.2 the modular function with respect to the Fubini-Study volume form is

$$
f_{F S}=\sum_{i=1}^{n} h_{i}
$$

Lemma 6.1. The $2 n$ hamiltonians $F=\left\{l^{*}\left(c_{i}\right), h_{i}\right\}_{i=1}^{n}$, , are in involution.

Proof. Indeed, the $c_{i}$ 's are in involution with respect to $\pi_{t}$ for all $t$ due to the bihamiltonian nature of the integrable model and $l^{*}$ is a Poisson morphism; moreover for each $[g \gamma] \in \mathcal{G}\left(\mathbb{C} P_{n}, \pi_{t}\right)$ we compute that

$$
\left\{l^{*}\left(c_{i}\right), h_{j}\right\}([g \gamma])=\left.\frac{d}{d t} l^{*}\left(c_{i}\right)\left(g \gamma e^{t H_{j}}\right)\right|_{t=0}=\left.\frac{d}{d t} c_{i}\left(g e^{t H_{j}}\right)\right|_{t=0}=0
$$

where we used the $\mathbb{T}^{n}$-invariance of $c$ and the fact that $\mathbb{T}^{n}$ normalizes $S B(n+1)$.

It is important to compute the following formula.

Lemma 6.2. On $[g \gamma] \in \mathcal{G}\left(\mathbb{C} P_{n}, \pi_{t}\right)$, we have that $h_{k}[g \gamma]=\operatorname{det}_{k} \gamma$, where $\operatorname{det}_{k}$ denotes the determinant of the first $k \times k$ minor.

Proof. We have that

$$
h_{k}[g \gamma]=\operatorname{Im} \operatorname{Tr}\left(H_{k} \log \operatorname{pr}_{A_{n+1}} \gamma\right)=\sum_{i=1}^{k} \log \gamma_{i i}-\frac{k}{n+1} \sum_{i=1}^{n+1} \log \gamma_{i i}=\log \operatorname{det}_{k} \gamma
$$

where we used the fact that $\operatorname{det} \gamma=1$. 
Let us first show that the integrable system is multiplicative. For each $(c, h) \in$ $\mathbb{R}^{n} \times \mathbb{R}^{n}$, let $L_{c h}=\left\{[g \gamma] \in \mathcal{G}\left(\mathbb{C} P_{n}, \pi_{t}\right) \mid c(g)=c, h(\gamma)=h\right\}$ denote the contour level set. The proof that $L_{c h}$ is connected is postponed to Proposition $\left.6.2 i i\right)$.

It is easy to check that the map that sends $(c, h) \in \mathbb{R}^{n} \times \mathbb{R}^{n}$ to

$$
r(c, h)_{i}=1-t+e^{-h_{i}}\left(c_{i}+t-1\right),
$$

defines a $\mathbb{R}^{n}$-action. Let $\mathbb{R}^{n} \rtimes \mathbb{R}^{n}$ denote the action groupoid and let $\left.\mathbb{R}^{n} \rtimes \mathbb{R}^{n}\right|_{\Delta_{n}}$ denote the action groupoid restricted to the standard simplex $\Delta_{n} \subset \mathbb{R}^{n}$.

Proposition 6.1. The space of contour level sets $\mathcal{G}_{F}\left(\mathbb{C} P_{n}, \pi_{t}\right)$ inherits the structure of topological groupoid. For $t \in(0,1)$, it is equivalent to the following wide subgroupoid of $\left.\mathbb{R}^{n} \rtimes \mathbb{R}^{n}\right|_{\Delta_{n}}$,

$$
\mathcal{G}_{F}\left(\mathbb{C} P_{n}, \pi_{t}\right)=\left\{\left.(c, h) \in \mathbb{R}^{n} \rtimes \mathbb{R}^{n}\right|_{\Delta_{n}} \mid c_{i}=c_{i+1}=1-t \Longrightarrow h_{i}=h_{i+1}\right\} .
$$

If $t \in\{0,1\}$ then it is equivalent to the wide subgroupoid

$$
\mathcal{G}_{F}\left(\mathbb{C} P_{n}, \pi_{t}\right)=\left\{\left.(c, h) \in \mathbb{R}^{n} \rtimes \mathbb{R}^{n}\right|_{\Delta_{n}} \mid c_{i}=1-t \Longrightarrow h_{i}=0\right\} .
$$

As a consequence, the modular function with respect to the Fubini-Study volume form is multiplicatively integrable.

Proof. Let the space of units $\left(\mathcal{G}_{F}\right)_{0}$ be the contour level sets of $c$. Let $[g \gamma] \in$ $L_{c h}$; we define $l\left(L_{c h}\right)=L_{c}$ and $r\left(L_{c h}\right)=L_{c(r[g \gamma])}$. We want to show that this map is well defined and coincides with (23). If $c_{i}=1-t$, then also $c_{i}(r([g \gamma]))=$ $1-t$, since fixing $c_{i}=1-t$ defines the Poisson submanifold $P_{i}(t)$, see (12) and (20). If $c_{k}(r(g \gamma)) \neq 1-t$ then the corresponding generator of the Cartan is hamiltonian with respect to $\pi_{t}$ on every connected component of $c_{k} \neq 1-t$ with hamiltonian $b_{k}$, where $b_{k}$ is the action variable defined in Proposition 5.1. As a consequence, when restricted to this component $h_{k}$ has the following local exact form in groupoid cohomology, i.e. $h_{k}=l^{*}\left(b_{k}\right)-r^{*}\left(b_{k}\right)$, that is, even though $b_{k}$ is not globally defined, the difference $l^{*}\left(b_{k}\right)-r^{*}\left(b_{k}\right)$ is. We then get

$$
h_{k}[g \gamma]=\log \frac{c_{k}+t-1}{r(c, h)_{k}+t-1}
$$

i.e. formula (23). Since $h$ is a groupoid cocycle, the map that sends $L_{c h}$ to $(c, h) \in$ $\left.\mathbb{R}^{n} \rtimes \mathbb{R}^{n}\right|_{\Delta_{n}}$ respects the groupoid multiplication. We have to characterize its image. If $c_{k}=1-t$ and $[g \gamma] \in L_{c h}$ then we can choose $g \in S(U(k) \times U(n+1-k))$ so that $\gamma=g^{-1} \lambda g^{\gamma}$ for some $\lambda \in U_{t}(n)^{\perp}$, see (17). Since $S(U(k) \times U(n+1-k))$ is a Poisson subgroup, and so it is invariant with respect to the dressing action, we can write in blocks

$$
g=\left(\begin{array}{cc}
g_{11} & 0 \\
0 & g_{22}
\end{array}\right), \quad \lambda=\left(\begin{array}{cc}
\lambda_{11} & \lambda_{12} \\
0 & \lambda_{22}
\end{array}\right), \quad g^{\gamma}=\left(\begin{array}{cc}
g_{11}^{\gamma} & 0 \\
0 & g_{22}^{\gamma}
\end{array}\right)
$$


We can then compute

$$
\exp h_{k}[g \gamma]=\operatorname{det}_{k}(\gamma)=\operatorname{det}\left(g_{11}^{-1}\right) \operatorname{det}\left(\lambda_{11}\right) \operatorname{det}\left(g_{11}^{\gamma}\right)=\operatorname{det}\left(\lambda_{11}\right)=\operatorname{det}_{k}(\lambda),
$$

since the det is invariant under the dressing action in $U(k)$. Let us recall that $\mathfrak{u}_{t}(n)^{\perp}=\operatorname{Ad}_{\sigma_{t}}^{*} \mathfrak{u}_{0}(n)^{\perp}$ (see (77)), so that we can write $\lambda=\exp \operatorname{Ad}_{\sigma_{t}}^{*} \xi$ for $\xi \in$ $\mathfrak{u}_{0}(n)^{\perp}$, i.e. $\xi_{i j} \neq 0$ only if $i=1, j>1$. A direct computation shows that

$$
\operatorname{pr}_{A_{n+1}}\left(\operatorname{Ad}_{\sigma_{t}}^{*} \xi\right)=\sqrt{t(1-t)} \xi_{1, n+1} \operatorname{diag}(1,0, \ldots,-1)
$$

so that we see from [18 that $h_{k}[g \gamma]=\sqrt{t(1-t)} \xi_{1, n+1}$ and the result easily follows.

Remark 6.3. The orbits of $\mathcal{G}_{F}\left(\mathbb{C} P_{n}, \pi_{t}\right)$ correspond to the decomposition of $\mathbb{C} P_{n}$ in regular Poisson strata given in (9) for $t=0,1$ and in Proposition 4.1 for $t \in(0,1)$. Moreover, the restrictions on $h$ reflect the fact that when $t \in(0,1)$ on each non maximal stratum the Cartan vector fields which are transversal to the symplectic leaves collapse to a single $\mathbb{S}^{1}$ generator, while for $t=0,1$ they are all zero.

\subsection{The Bohr-Sommerfeld groupoid $\mathcal{G}_{F}^{b s}\left(\mathbb{C} P_{n}, \pi_{t}\right)$}

In order to compute the Bohr-Sommerfeld leaves we need to show that $L_{c h}$ is connected and describe $H_{1}\left(L_{c h}, \mathbb{Z}\right)$. It is obvious that for each $c \in \Delta_{n} L_{c}$ is a $\mathbb{T}^{n}$-orbit.

It is easy to produce 1-cycles in $L_{c h}$. The hamiltonian flux of $h_{j}, j=1 \ldots n$ and of $l^{*}\left(b_{j}\right)$ for $c_{j} \neq 1-t$ define a set of 1-cycles in $L_{c h}$. Indeed, fix $[g \gamma] \in L_{c h}$; $\alpha_{j}(t)=\left[g \gamma e^{t H_{j}}\right]$, for $j=1, \ldots n$, is the flux of $h_{j}$. Let us compute the flux of $l^{*}\left(b_{j}\right)$, for $c_{j} \neq 1-t$, and show that it is periodic.

Lemma 6.4. The hamiltonian flux of $l^{*}\left(b_{j}\right)$ passing through $[g \gamma] \in L_{c h}$ is given by

$$
\beta_{j}(t)=\left[g e^{B_{H_{j}}[g] t} \gamma\right]
$$

where

$$
B_{H_{j}}[g]=H_{j}+\sum_{a} \ell_{T_{a}}\left(b_{j}\right)([g]) T^{a} \in \mathfrak{s l}(n+1, \mathbb{C}),
$$

for some basis $\left\{T_{a}\right\}$ of $\mathfrak{s u}(n+1)$ and the dual basis $\left\{T^{a}\right\}$ of $\mathfrak{s} \mathfrak{b}(n+1)$. Moreover, $\beta_{j}(t)=\beta_{j}(t+2 \pi)$ for each $t$. 
Proof. Let $\beta_{j}(t)=\left[g_{j}(t) \gamma_{j}(t)\right]$ be the hamiltonian flux of $l^{*}\left(b_{j}\right)$. Since $l\left(\beta_{j}(t)\right)=\left[g e^{t H_{j}}\right]$ then $\left[g_{j}(t) \gamma_{j}(t)\right]=\left[g e^{t H_{j}} \gamma_{j}(t)\right]$. By using the definition of the Poisson tensor (21), especially the third term thereof that entangles $G$ and $G^{*}$, we get that

$$
\dot{\gamma}_{j}(t)=\left(\sum_{a} \ell_{T_{a}}\left(b_{j}\right)\left[g e^{t H_{j}}\right] T^{a}\right) \gamma_{j}(t)=e^{-t H_{j}}\left(\sum_{a} \ell_{T_{a}}\left(b_{j}\right)[g] T^{a}\right) e^{t H_{j}} \gamma_{j}(t),
$$

note that in the second equality we used the equivariance of the momentum map, which can be seen explicitly from

$$
\ell_{T_{a}}\left(c_{j}\right)([g])=\operatorname{Tr}\left[g^{-1} \lambda g\left[T_{a}, H_{j}\right]\right],
$$

where $\lambda$ is the weight of the fundamental representation of $S U(n+1)$.

The solution to the equation (24) is

$$
\gamma_{j}(t)=e^{-t H_{j}} e^{t B_{H_{j}}[g]} \gamma .
$$

We just have to show that $\beta_{j}$ is periodic. It is a direct computation to obtain a block matrix form for $B_{H_{j}}[g]$ : choose the generators of the Lie algebra of $N_{n+1} \subset S B(n+1, \mathbb{C})$ as $\left(E^{i j}\right)_{k l}=\delta_{i k} \delta_{j l}$ and $i E^{i j}$ with $i<j$, and the dual generators $i\left(E^{i j}+E^{j i}\right)$ and $\left(E^{i j}-E^{j i}\right)$. For $k, l<j$ or $k, l>j$ the latter commute with $H_{j}$, and hence from (25) the corresponding blocks of the matrix $\ell_{T_{a}}\left(c_{j}\right)([g])$ vanishes, the blocks that remain are

$$
B_{H_{j}}=\frac{i}{n+1}\left(\begin{array}{cc}
(n+1-k) \mathrm{id}_{k} & A \\
0 & -k \operatorname{id}_{n+1-k}
\end{array}\right) .
$$

It can be diagonalized to $H_{j}$ with the similarity transformation by

$$
\left(\begin{array}{cc}
1 & A /(n+1) \\
0 & 1
\end{array}\right)
$$

The periodicity of $\beta_{j}$ then follows from the observation that $\exp 2 \pi H_{j} \in \mathbb{Z}_{n} \subset$ $U_{t}(n)$.

We are going to show that $\left\{\alpha_{j}, \beta_{k}\right\}$ generate $H_{1}\left(L_{c h}, \mathbb{Z}\right)$. We will use arguments based on the use of the homotopy long exact sequence associated to a fibration. We need to introduce some definitions and preliminary results.

Let $x \sim_{t} y$ denote that $x, y \in \mathbb{C} P_{n}$ live in the same symplectic leaf of $\pi_{t}$. Let $\tilde{L}_{c h}=\left\{(x, y) \in L_{c} \times L_{r(c, h)} \mid x \sim_{t} y\right\} \subset L_{c} \times L_{r(c, h)}$; we have clearly that

$$
(l \times r): L_{c h} \rightarrow \tilde{L}_{c h} .
$$

Lemma 6.5. Let $x \in L_{c}$ and let $\mathbb{T}_{x}=\left\{s \in \mathbb{T}^{n} \mid x s \sim_{t} x\right\}$, Then we see that 
i) For each $x, y \in L_{c}, \mathbb{T}_{x}=\mathbb{T}_{y} \equiv \mathbb{T}_{c}$.

ii) For each $x \in L_{c}, T_{x}\left(x \mathbb{T}_{c}\right)=\left\langle\sigma_{H_{i}}(x), c_{i} \neq 1-t\right\rangle$.

iii) The hamiltonian flux of $l^{*}\left(b_{j}\right)$, for $c_{j} \neq 1-t$, described in Lemma 6.4 defines an action of $\mathbb{T}_{c}$ on $L_{c h}$ that commutes with the $\mathbb{T}^{n}$-action.

iv) $\mathbb{T}_{c} \times \mathbb{T}^{n}$ acts transitively on $\tilde{L}_{c h}$ as $(x, y)(s, k)=(x s k, y k)$, where $(x, y) \in$ $\tilde{L}_{c h},(s, k) \in \mathbb{T}_{c} \times \mathbb{T}^{n}$; the map (27) intertwines the actions.

Proof. $i$ ) Since the action of the Cartan is by Poisson diffeomorphisms, its tangent action commutes with the sharp map, so that if $x \sim_{t} y$ then $x \tau \sim_{t} y \tau$ for any $\tau \in \mathbb{T}^{n}$. Let $x \in L_{c}$ and $s \in \mathbb{T}_{x}$; let $y=x \tau$, for some $\tau \in \mathbb{T}^{n}$. We have that $x \sim_{t} x s$ implies $y \sim_{t}$ ys so that $s \in \mathbb{T}_{y}$.

ii) Let $x \in L_{c}$ and let $\mathcal{S}_{x}$ be the symplectic leaf through $x$. If $c_{i} \neq 1-t$ then $\sigma_{H_{i}}$ is locally hamiltonian and clearly preserves the leaf. Let $c_{i}=1-t$ and let $\sigma_{H_{i}}(x) \neq 0$ so that $d \phi_{i}(x)$ is defined and non zero. Clearly $\left\langle\sigma_{H_{i}}(x), d \phi_{i}(x)\right\rangle=1$. We can choose an open $U_{x}$ containing $x$ such that the points $y$ such that $d \phi_{i}(y)$ is defined and $c_{i}(y) \neq 1-t$ are dense. For such $y, \pi_{t}\left(d \phi_{i}(y)\right)=\partial / \partial_{b_{i}}=\left(c_{i}-1+\right.$ t) $\partial / \partial_{c_{i}}$ that extends to 0 in $x$. So we have that $d \phi_{i}(x) \in N_{x}^{*} \mathcal{S}_{x}$ and $\sigma_{H_{i}}(x) \notin T_{x} \mathcal{S}_{x}$.

iii) This is just a rephrasing of Lemma 6.4.

$i v)$ Let us first show transitivity. Let $(x, y),\left(x_{0}, y_{0}\right) \in \tilde{L}_{c h}$; then we know that $(x, y)=\left(x_{0} k_{1}, y_{0} k_{2}\right)=\left(x_{0} k_{1} k_{2}^{-1}, y_{0}\right)\left(1, k_{2}\right)$ for $k_{1}, k_{2} \in \mathbb{T}^{n}$, so that $x_{0} k_{1} k_{2}^{-1} \sim$ $y_{0} \sim x_{0}$ so that $k_{1} k_{2}^{-1} \in \mathbb{T}_{c}$ and $(x, y)=\left(x_{0}, y_{0}\right)\left(k_{1} k_{2}^{-1}, k_{2}\right)$. It is a trivial fact that $l \times r$ intertwines the $\mathbb{T}^{n}$-action; the same property with respect to $\mathbb{T}_{c}$ follows from the properties of the hamiltonian vector fields of $l^{*}\left(b_{j}\right)$.

For each $[g] \in \mathbb{C} P_{n}$ let us denote $N_{n+1}[g]=\left\{n \in N_{n+1} \mid{ }^{g} n \in U_{t}(n)^{\perp},\left[g^{n}\right]=\right.$ $[g]\}=g^{-1} U_{t}(n)^{\perp} U_{t}(n) g \cap N_{n+1}$; it is clearly a subgroup of $N_{n+1}$. Next we shall show that this subgroup is of exponential type (and hence contractible) and that two such subgroups are isomorphic if the base points live in the same $\mathbb{T}^{n}$-orbit.

Lemma 6.6. $\quad$ i) $N_{n+1}[g] \subset N_{n+1}$ is connected and then of exponential type.

ii) For $s \in \mathbb{T}^{n}$, the map $n \rightarrow s^{-1} n$ is a group isomorphism from $N_{n+1}[g s] \rightarrow$ $N_{n+1}[g]$.

Proof. i) We can easily see that $N_{n+1}[g]={ }_{[g]} \mathcal{G}\left(\mathbb{C} P_{n}, \pi_{t}\right)_{[g]} \cap N_{n+1}$. From Lemma 1 in [10, we know that

$$
\pi_{0}\left({ }_{[g]} \mathcal{G}\left(\mathbb{C} P_{n}, \pi_{t}\right)_{[g]}\right)=\pi_{1}\left(\mathcal{S}_{[g]}\right),
$$

where $\mathcal{S}_{[g]}$ is the symplectic leaf through $[g]$. Since in our case the symplectic leaves are all contractible (see Lemma 4.2), we conclude that $N_{n+1}[g]$ is the intersection of two connected subgroups of $S B(n+1, \mathbb{C})$; since connected subgroups of 
an exponential group are of exponential type and are closed under the intersection (see [24] pg.9) we get the result. Point ii) is obtained by direct computation.

Let $N_{c}$ denote the group bundle over $L_{c}$ with fibre $N_{n+1}[g]$ over $[g] \in L_{c}$ and let $p: N_{c} \rightarrow L_{c}$ denote the projection; it acts on $L_{c h}$ with anchor $l: L_{c h} \rightarrow L_{c}$ and action $a: N_{c p} \times_{l} L_{c h} \rightarrow L_{c h}$ given by

$$
a(n,[g \gamma])=[g n \gamma]
$$

This action preserves the fibres of (27).

Proposition 6.2. $\quad$ i) The map (27) defines a fibre bundle and the groupoid action of $N_{c}$ on $L_{c h} \rightarrow \tilde{L}_{c h}$

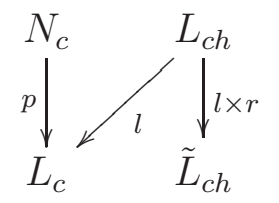

defines a principal groupoid bundle.

ii) $L_{c h}$ is connected and $H_{1}\left(L_{c h}, \mathbb{Z}\right)$ is generated by $\left\langle\alpha_{j}, \beta_{k}\right\rangle$.

Proof. $i$ ) Let us show first that we have a groupoid principal bundle according to definition in section 5.7 of [21]. The map $l \times r: L_{c h} \rightarrow \tilde{L}_{c h}$ is a surjective submersion as a consequence of Lemma 6.5 (iv). Next we have to show that the map

$$
\left(a, \operatorname{pr}_{2}\right): N_{c} L_{c} \times_{L_{c}} L_{c h} \rightarrow L_{c h} \tilde{L}_{c h} \times_{\tilde{L}_{c h}} L_{c h}, \quad(n,[g \gamma]) \rightarrow([g n \gamma],[g \gamma])
$$

is a diffeomorphism. Let $([\tilde{g} \tilde{\gamma}],[g \gamma]) \in L_{c h} \tilde{L}_{c h} \times_{\tilde{L}_{c h}} L_{c h}$, i.e. $(l \times r)[g \gamma]=(l \times r)[\tilde{g} \tilde{\gamma}]$. Then we see that $[g]=[\tilde{g}]$ and $g^{\tilde{\gamma}}=h g^{\gamma}$ for $h \in U_{t}(n)$. Define $n=\tilde{\gamma} \gamma^{-1} \in N_{n+1}$. Since we also have that ${ }^{g} \gamma,{ }^{g} \tilde{\gamma} \in U_{t}(n)^{\perp}$ and recalling that the dressing action of $U_{t}(n)$ preserves $U_{t}(n)^{\perp}$ (and viceversa), one shows that

$$
\begin{aligned}
{ }^{g} n & ={ }^{g} \tilde{\gamma}{ }^{g^{\tilde{\gamma}}} \gamma^{-1}={ }^{g} \tilde{\gamma}^{h}\left(g^{\gamma} \gamma^{-1}\right)={ }^{g} \tilde{\gamma}^{h}\left({ }^{g} \gamma\right)^{-1} \in U_{t}(n)^{\perp} \\
g^{n} & =g^{\tilde{\gamma} \gamma^{-1}}=\left(h g^{\gamma}\right)^{\gamma^{-1}}=h^{\left({ }^{g} \gamma\right)^{-1}} g=h^{\prime} g
\end{aligned}
$$

for $h, h^{\prime} \in U_{t}(n)$. We conclude that $n \in N_{n+1}[g]$ and the map $([\tilde{g} \tilde{\gamma}][g \gamma]) \rightarrow$ $(n,[g \gamma])$ defines an inverse of $\left(a, \mathrm{pr}_{2}\right)$. The map $\delta_{N_{c}}: L_{c h} \tilde{L}_{c h} \times_{\tilde{L}_{c h}} L_{c h} \rightarrow N_{c}$, $([\tilde{g} \tilde{\gamma}][g \gamma]) \rightarrow \tilde{\gamma} \gamma^{-1}$, is called the division map.

Due to Lemma 6.5 iv), $\tilde{L}_{c h}$ is homeomorphic to $\mathbb{T}_{c} \times \mathbb{T}^{n} / T$ for $T$ being the stability subgroup. Let us fix $\left[g_{0} \gamma_{0}\right] \in L_{c h}$ and let $\left(x_{0}, y_{0}\right)=(l \times r)\left[g_{0} \gamma_{0}\right]$ and $V_{0}$ an open containing $\left(x_{0}, y_{0}\right)$ and admitting a section $(s, k): V_{0} \rightarrow \mathbb{T}_{c} \times \mathbb{T}^{n}$ 
such that $(x, y)=\left(x_{0}, y_{0}\right)(s(x, y), k(x, y))$ for each $(x, y) \in V_{0}$. Let $\left.[g \gamma] \in L_{c h}\right|_{V_{0}}$ and $(x, y)=(l \times r)[g \gamma]$; then $[g \gamma](s(x, y), k(x, y))^{-1} \in(l \times r)^{-1}\left(x_{0}, y_{0}\right)$ and $[g \gamma] \rightarrow \delta_{N_{c}}\left(\left[g_{0}, \gamma_{0}\right],[g \gamma](s(x, y), k(x, y))^{-1}\right) \in N_{\left[g_{0}\right]}$ gives the local trivialization.

ii) We apply the long exact homotopy sequence to the fibre bundle (27). Since the fibre is contractible we get that $\pi_{k}\left(L_{c h}\right)=\pi_{k}\left(\tilde{L}_{c h}\right)$, in particular $L_{c h}$ is connected. The same homotopy sequence applied to $T \rightarrow \mathbb{T}_{c} \times \mathbb{T}^{n} \rightarrow \tilde{L}_{c h}$, since $T$ is connected, shows that the map $\pi_{1}\left(\mathbb{T}_{c} \times \mathbb{T}^{n}\right) \rightarrow \pi_{1}\left(\tilde{L}_{c h}\right)=\pi_{1}\left(L_{c h}\right)$, sending the generators of $\mathbb{T}_{c} \times \mathbb{T}^{n}$ to $\left\{\alpha_{j}, \beta_{k}\right\}$, is surjective. The statement on homology easily follows.

We are now ready for the computation of the groupoid of Bohr-Sommerfeld leaves. From the discussion at the end of Section 3, we know that the symplectic form $\Omega_{\mathcal{G}}$ is exact. In the following Lemma we show that there exists a primitive $\Theta_{\mathcal{G}}$, i.e. a one form $\Theta_{\mathcal{G}}$ satisfying $d \Theta_{\mathcal{G}}=\Omega_{\mathcal{G}}$, adapted to the momentum map $h$ of the action of the Cartan $\mathbb{T}^{n}$. Remark that the fact that $h$ is an $\mathbb{R}^{n}$-valued cocycle plays a role.

Lemma 6.7. There exists a primitive $\Theta_{\mathcal{G}}$ of the symplectic form $\Omega_{\mathcal{G}}$ such that for each $H \in \mathfrak{t}_{n}$

$$
\iota_{\sigma_{H}} \Theta_{\mathcal{G}}=-h_{H} \quad,
$$

where $\sigma_{H}$ is the fundamental vector field corresponding to $H$.

Proof. Since $\mathbb{T}^{n}$ is compact, by averaging, we can always find a $\mathbb{T}^{n}$-invariant primitive $\Theta_{\mathcal{G}}$, i.e. $L_{\sigma_{H}} \Theta_{\mathcal{G}}=0$. By using the definition of the momentum map we easily see that

$$
\iota_{\sigma_{H}} \Theta_{\mathcal{G}}+h_{H}=a_{H} \in \mathbb{R} .
$$

Since the submanifolds of units $\mathcal{G}_{0}=\mathbb{C} P_{n}$ is lagrangian then $\left.\Theta_{\mathcal{G}}\right|_{\mathcal{G}_{0}}$ is closed and since $H^{1}\left(\mathbb{C} P_{n}\right)=0$ then $\left.\Theta_{\mathcal{G}}\right|_{\mathcal{G}_{0}}$ is exact. The $\mathbb{T}^{n}$-action on $\mathcal{G}\left(\mathbb{C} P_{n}, \pi_{t}\right)$ preserves $\mathcal{G}_{0}$; moreover for any orbit $\gamma_{H} \subset \mathbb{C} P_{n}$ of $\sigma_{H}$ we have that $\left.h_{H}\right|_{\gamma}=0$ because $h_{H}$, being an $\mathbb{R}$-valued cocycle, is zero on the units. We then conclude that

$$
\int_{\gamma_{H}} \Theta_{\mathcal{G}}=a_{H}=0
$$

From now on, $\Theta_{\mathcal{G}}$ will denote a fixed primitive satisfying the condition of Lemma 6.7.

Theorem 6.3. For $\hbar \in \mathbb{R}$, the groupoid of Bohr-Sommerfeld leaves is the following subgroupoid of $\mathcal{G}_{F}\left(\mathbb{C} P_{n}, \pi_{t}\right)$ described in Proposition 6.1

$$
\mathcal{G}_{F}^{b s}\left(\mathbb{C} P_{n}, \pi_{t}\right)=\left\{(c, h) \in \mathcal{G}_{F}\left(\mathbb{C} P_{n}, \pi_{t}\right)|\log | c_{k}-1+t \mid \in \hbar \mathbb{Z}, h_{k} \in \hbar \mathbb{Z}\right\} \quad .
$$


It is an ètale groupoid and admits a unique left Haar system for all $t \in[0,1]$. The generators of the Cartan subgroup are quantized to the groupoid cocycles $h_{i}(c, h)=h_{i}$; in particular the modular cocycle reads

$$
f_{F S}(c, h)=\sum_{i=1}^{n} h_{i} \in \hbar \mathbb{Z}
$$

Proof. Let $L_{c h} \in \mathcal{G}_{F}\left(\mathbb{C} P_{n}, \pi_{t}\right)$ and let $c_{k} \neq 1-t$. Let $\Theta_{\mathcal{G}}$ be a primitive of $\Omega_{\mathcal{G}}$ as in Lemma 6.7, we have to pair it with the generators $\left\langle\alpha_{j}, \beta_{k}\right\rangle$ of $H_{1}\left(L_{c h}, \mathbb{Z}\right)$. We then compute the Bohr-Sommerfeld conditions as

$\int_{\beta_{k}} \Theta_{\mathcal{G}}=\left.\int_{\tilde{\beta}_{k}} \Omega_{\mathcal{G}}\right|_{\mathcal{G}_{[g]}}=\int_{\tilde{\beta}_{k}} l^{*} \omega_{\mathcal{S}_{[g]}}=\int_{l\left(\tilde{\beta}_{k}\right)} \omega_{\mathcal{S}_{[g]}}=b_{k} 2 \pi=2 \pi \log \left|c_{k}-1+t\right| \in 2 \pi \hbar \mathbb{Z}$,

where $\tilde{\beta}_{k}: \mathbb{D} \rightarrow \mathcal{G}\left(\mathbb{C} P_{n}, \pi_{t}\right)_{[g]}$ extends $\beta_{k}$ to the disk and in the second equality we use the fact that $\left.\Omega\right|_{\mathcal{G}_{[g]}}=l^{*} \omega_{\mathcal{S}_{[g]}}$. Since $\iota_{\sigma_{H}} \Theta_{\mathcal{G}}=-h_{H}$, for each $H \in \mathfrak{t}_{n}$, we easily compute

$$
\int_{\alpha_{j}} \Theta_{\mathcal{G}}=\int_{0}^{2 \pi} \iota_{\sigma_{H_{j}}} \Theta_{\mathcal{G}} d t=-2 \pi h_{j} \in 2 \pi \hbar \mathbb{Z}
$$

The fact that the set of Bohr-Sommerfeld leaves $\mathcal{G}_{F}^{b s}\left(\mathbb{C} P_{n}, \pi_{t}\right)$ is a subgroupoid is a straightforward computation.

Let $(c, h) \in \mathcal{G}_{F}^{b s}\left(\mathbb{C} P_{n}, \pi_{t}\right)$ and let $I_{\epsilon}(c, h)=f_{F S}^{-1}\left(I_{\epsilon}\left(f_{F S}(c, h)\right)\right)$ the open neighborhood of $f_{F S}(c, h)$ of radius $\epsilon>0$. It is clear that for $\epsilon<1, I_{\epsilon}(c, h)=\left\{\left(c^{\prime}, h\right) \in\right.$ $\left.\mathcal{G}_{F}^{b s}\left(\mathbb{C} P_{n}, \pi_{t}\right)\right\}$ so that $l$ is a local homeomorphism. By applying Proposition 2.2 we conclude that $\mathcal{G}_{F}^{b s}\left(\mathbb{C} P_{n}, \pi_{t}\right)$ is étale and admits a unique left Haar system, the counting measure on the $l$-fibres.

The space of units of $\mathcal{G}_{F}^{b s}\left(\mathbb{C} P_{n}, \pi_{t}\right)$ is the following subset of the standard simplex

$$
\Delta_{n}^{\mathbb{Z}}(t)=\left\{c \in \Delta_{n} \mid c_{k}=1-t \pm e^{-\hbar n_{k}}, n_{k} \in \mathbb{Z}\right\} .
$$

For $\hbar>0$, the quasi invariant measure on $\Delta_{n}^{\mathbb{Z}}(t)$ associated to $f_{F S}$ is computed as

$$
\mu_{F S}(c)=\operatorname{det}\left(J_{\lambda}(c)+t\right)=\Pi_{k}\left|c_{k}-1+t\right|=\exp \left(-\hbar \sum_{k=1}^{n} n_{k}\right) .
$$

The components of $\mathcal{G}_{F}^{b s}\left(\mathbb{C} P_{n}, \pi_{t}\right)$ are labeled by the couples $(r, s)$ of non negative integers such that $r+s \leq n$. Indeed the component $\mathcal{G}_{F}^{b s}\left(\mathbb{C} P_{n}, \pi_{t}\right)^{(r, s)}$ is the restriction of $\mathcal{G}_{F}^{b s}\left(\mathbb{C} P_{n}, \pi_{t}\right)$ to $c_{k}=1-t$ for $r<k \leq n-s$. Let us describe this component more explicitly. Let $\mathbb{Z}^{n}$ act by translation on $\overline{\mathbb{Z}}^{n}$, where $\overline{\mathbb{Z}}=\mathbb{Z} \cup\{\infty\}$ 

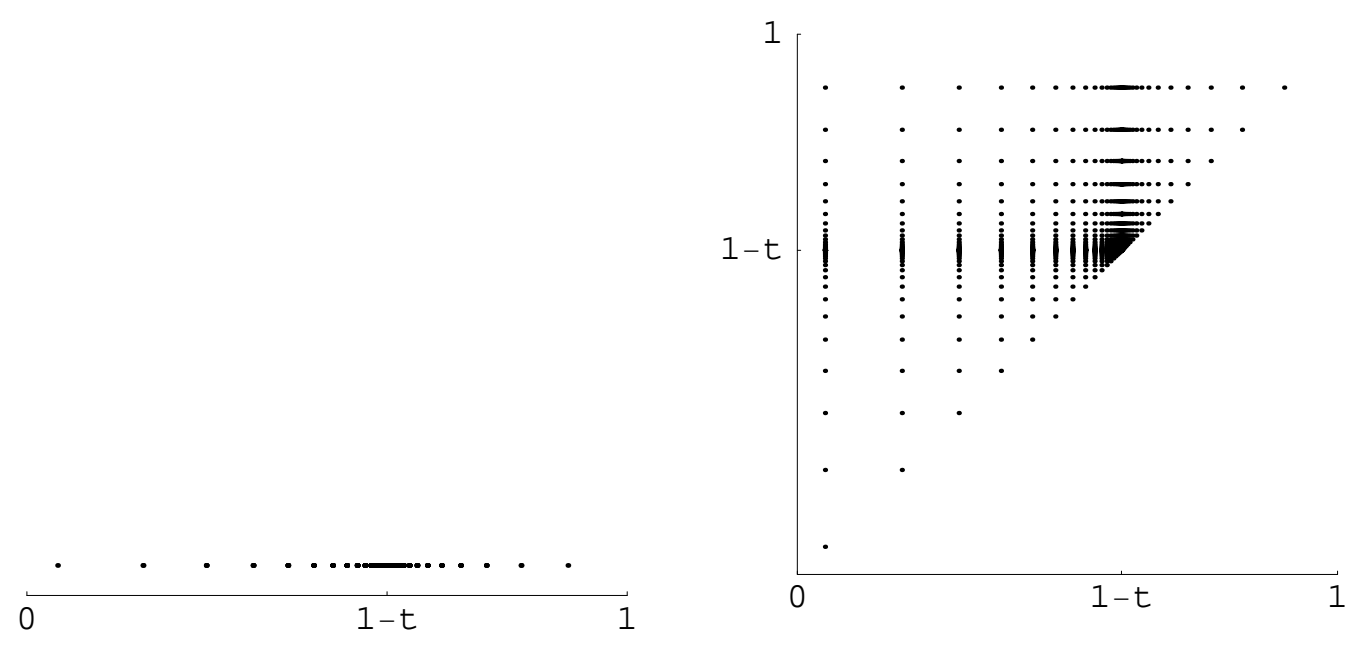

Figure 1: $\Delta_{1}^{\mathbb{Z}}(t)$ and $\Delta_{2}^{\mathbb{Z}}(t)$

be the one point compactification. Let $\overline{\mathbb{Z}}^{n} \rtimes \mathbb{Z}^{n}$ be the action groupoid. Let $\Delta_{r, s}^{\mathbb{Z}}(t) \subset \overline{\mathbb{Z}}^{n}$ be defined as

$$
\begin{aligned}
\left\{(m, \infty \ldots \infty, n) \in \overline{\mathbb{Z}}^{r} \times \overline{\mathbb{Z}}^{n-r-s} \times \overline{\mathbb{Z}}^{s} \mid \quad\right. & -\frac{1}{\hbar} \log (1-t) \leq m_{i} \leq m_{i+1}, \\
& \left.n_{i} \geq n_{i+1} \geq-\frac{1}{\hbar} \log t\right\} .
\end{aligned}
$$

Note that $\Delta_{r, s}^{\mathbb{Z}}(t)$ corresponds to the stratum $\mathbb{C} P_{n}^{(r, s)}$ of dimension $2(r+s)$ of $\left(\mathbb{C} P_{n}, \pi_{t}\right)$ described in Proposition 4.1 for $t \in(0,1)$. Indeed one can check that (16) can be restated as

$$
c_{r}<1-t=c_{r+1}=c_{r+2}=\ldots=c_{n-s}<c_{n-s+1},
$$

whose $B S$ leaves are exactly those in $\Delta_{r, s}^{\mathbb{Z}}(t)$. Analogously $\Delta_{r, 0}(0)$ corresponds to the $2 r$-dimensional Bruhat cell for $t=0$.

For $t \in(0,1)$, the component $\mathcal{G}_{F}^{b s}\left(\mathbb{C} P_{n}, \pi_{t}\right)^{(r, s)}$ is isomorphic to the following subgroupoid of the action groupoid $\left.\overline{\mathbb{Z}}^{n} \rtimes \mathbb{Z}^{n}\right|_{\Delta_{r, s}^{Z}}$

$$
\left\{(q, p) \in \overline{\mathbb{Z}}^{n} \times\left.\mathbb{Z}^{n}\right|_{\Delta_{r, s}^{Z}} \mid q_{i}=q_{i+1}=\infty \Longrightarrow p_{i}=p_{i+1}\right\} .
$$

If $t=0$ there are only components like $\mathcal{G}_{F}^{b s}\left(\mathbb{C} P_{n}, \pi_{t}\right)^{(r, 0)}=\mathcal{G}^{b s,(r, 0)}$ that is isomorphic to the following subgroupoid of the action groupoid $\left.\overline{\mathbb{Z}}^{n} \rtimes \mathbb{Z}^{n}\right|_{\Delta_{r, 0}^{Z}}$

$$
\left\{(q, p) \in \overline{\mathbb{Z}}^{n} \times\left.\mathbb{Z}^{n}\right|_{\Delta_{r, 0}^{Z}} \mid q_{i}=\infty \Longrightarrow p_{i}=0\right\} .
$$

The description for $t=1$ is analogous. 


\subsection{Quantization of Poisson morphisms}

We discuss in this Section the covariance of the correspondence that sends $\left(\mathbb{C} P_{n}, \pi_{t}\right)$ to the étale groupoid $\mathcal{G}_{F}^{b s}\left(\mathbb{C} P_{n}, \pi_{t}\right)$, established in Theorem 6.3, with respect to some of the Poisson isomorphisms that we discussed in previous Sections.

Let us first define the map $\psi: \overline{\mathbb{Z}}^{n} \rightarrow \overline{\mathbb{Z}}^{n}$ as $\psi(q)_{i}=q_{n+1-i}$. The following Lemma, proved by a direct check, shows that the Poisson isomorphism between $\left(\mathbb{C} P_{n}, \pi_{t}\right)$ and $\left(\mathbb{C} P_{n},-\pi_{1-t}\right)$ in Lemma 4.1 is quantized by a groupoid isomorphism.

Lemma 6.8. The map from $\in \mathcal{G}_{F}^{b s}\left(\mathbb{C} P_{n}, \pi_{t}\right)^{(r, s)}$ to $\mathcal{G}_{F}^{b s}\left(\mathbb{C} P_{n}, \pi_{1-t}\right)^{(s, r)}$ sending $(q, p)$ to $(\psi(q), \psi(p))$ extends to a groupoid isomorphism $\hat{\psi}: \mathcal{G}_{F}^{b s}\left(\mathbb{C} P_{n}, \pi_{t}\right) \rightarrow$ $\mathcal{G}_{F}^{b s}\left(\mathbb{C} P_{n},-\pi_{1-t}\right)$ for each $t \in[0,1]$.

We are then going to show that the Poisson embeddings

$$
i_{k}: P_{k}(t) \equiv p_{t}\left(S(U(k) \times U(n+1-k)) \rightarrow\left(\mathbb{C} P_{n}, \pi_{t}\right)\right.
$$

described in Section 4 are quantized to subgroupoids of $\mathcal{G}_{F}^{b s}\left(\mathbb{C} P_{n}, \pi_{t}\right)$. As a result we get at the same time the quantization of these Poisson structures and show the covariance of the quantization with respect to these Poisson morphisms.

We know from general results that $\left.\mathcal{G}\left(\mathbb{C} P_{n}, \pi_{t}\right)\right|_{P_{k}(t)}=\left\{[g \gamma] \mid c_{k}[g]=1-\right.$ $t\}$ is coisotropic and the symplectic groupoid integrating $P_{k}(t)$ is obtained as coisotropic reduction. The coisotropic foliation is generated by the hamiltonian flux of $c_{k}$

$$
\mathcal{G}\left(P_{k}(t), \pi_{t}\right)=\mathcal{G}\left(\mathbb{C} P_{n}, \pi_{t}\right) / /\left\{c_{k}=1-t\right\} \quad .
$$

The set of hamiltonians $F=\left\{c_{i}, i \neq k, h_{j}\right\}$ clearly descends to a set defining a multiplicative integrable model. All the analysis of the previous Section descends to the quotient, so that we get the following Proposition.

Proposition 6.4. For $t \in[0,1]$, the Poisson submanifold $P_{k}(t)$ is quantized by the subgroupoid

$$
\mathcal{G}_{F}^{b s}\left(P_{k}(t), \pi_{t}\right)=\left\{(c, h) \in \mathcal{G}_{F}^{b s}\left(\mathbb{C} P_{n}, \pi_{t}\right) \mid c_{k}=1-t\right\} \quad .
$$

By applying the contravariant functor assigning to a groupoid its $C^{*}$-algebra, we get a family of $C^{*}$-algebra short exact sequences

$$
0 \rightarrow \mathcal{I}_{k} \rightarrow C^{*}\left(\mathcal{G}_{F}^{b s}\left(\mathbb{C} P_{n}, \pi_{t}\right)\right) \rightarrow C^{*}\left(\mathcal{G}_{F}^{b s}\left(P_{k}(t), \pi_{t}\right)\right) \rightarrow 0
$$

It can be remarked that putting $c_{n-1}=1-t$ in $\mathcal{G}_{F}\left(P_{n}(t), \pi_{t}\right)$, and then $c_{n-2}$ and so on, we obtain a chain of subgroupoids, that corresponds to the chain of Poisson embeddings of spheres appearing as the right side of (14). 


\section{Quantum homogeneous spaces as groupoid $C^{*}$-algebras}

In this Section we will discuss how the quantization of the Poisson pencil and Poisson submanifolds that we described in Theorem 6.3 and Proposition 6.4 is related to quantum spaces appearing in quantum group theory.

The family of quantum homogeneous spaces $\mathbb{C} P_{n, q, t}$ was first introduced in [12], generalizing previous work by Podleś on $\mathbb{C} P_{1}$. In such paper a parametrized family of right coideal $*$-subalgebras of the Hopf algebra $\mathcal{A}\left(S U_{q}(n+1)\right)$ of representative functions was introduced. The algebra $\mathcal{A}\left(\mathbb{C} P_{n, q, t}\right)$ is then defined as the subalgebra of coinvariants and is characterized in terms of generators and relations: it is a noncommutative deformation of the algebra of polynomial functions on $\mathbb{C} P_{n}$ whose semiclassical limit gives the Poisson structure $\left(\mathbb{C} P_{n}, \pi_{t}\right)$. In a similar manner the odd-dimensional quantum spheres $\mathcal{A}\left(\mathbb{S}_{q}^{2 n-1}\right)$ had already been previously defined as right coideal *-subalgebras of $\mathcal{A}\left(S U_{q}(n+1)\right)$ in [30] as one of the first examples of quantum homogeneous spaces. It has to be remarked here that quantum odd spheres and standard complex projective spaces are quotients by quantum subgroups, while in the non standard case the quotient procedure requires the use of coisotropic quantum subgroups, in perfect agreement with what happens at the semiclassical level.

There is a canonical way to complete both these polynomial algebras to the $C^{*}$-algebra $C\left(\mathbb{C} P_{n, q, t}\right)$ and $\mathcal{C}\left(\mathbb{S}_{q}^{2 n-1}\right)$. The $C^{*}$-norm is defined as $\|a\|=$ $\sup _{\rho}\|\rho(a)\|$ of the operator norms among bounded $*$-representations $\rho$ of the polynomial algebra.

In 25] Sheu, relying on the representation theory of $\mathcal{A}\left(\mathbb{C} P_{n, q, t}\right)$, proved that such algebra is in fact, the groupoid $C^{*}$-algebra of a topological groupoid when $t=0,1$, and also, for arbitrary $t$ in the case $n=1$. In the more general case in which $n>1$ and $0<t<1$ his results were less conclusive though, basing on the same construction, he was able to show that $C\left(\mathbb{C} P_{n, q, t}\right)$ is contained in a groupoid $C^{*}$-algebra. On the other hand he was able to prove the existence of a short exact sequence of $C^{*}$-algebras $0 \rightarrow \mathcal{I} \rightarrow C\left(\mathbb{C} P_{n, q, t}\right) \rightarrow C\left(\mathbb{S}_{q}^{2 n-1}\right) \rightarrow 0$ relating the non standard quantum complex projective spaces to odd-dimensional quantum spheres.

We are going to show how our quantization of $\left(\mathbb{C} P_{n}, \pi_{t}\right)$, for $t \in[0,1]$, is related to these results. In the cases covered by Sheu's description like $\left(\mathbb{C} P_{n}, \pi_{0}\right)$ and $\left(P_{k}(t)=\mathbb{S}^{2 n-1}, \pi_{t}\right)$, for $k=1, n$, we will then establish that our quantization of the symplectic groupoid produces the same $C^{*}$-algebra of the quantum spaces obtained via quantum group techniques. We conjecture that this is also true for $\left(\mathbb{C} P_{n}, \pi_{t}\right)$ and $\left(P_{k}(t), \pi_{t}\right)$, for $t=(0,1)$ and $1<k<n$. 


\subsection{The standard $\mathbb{C} P_{n, q, 0}$}

In [25], it was shown that the $C^{*}$-algebra of standard quantum projective spaces is the $C^{*}$-algebra of a groupoid $\mathfrak{T}_{n}$ defined as follows. Let us consider the translation action of $\mathbb{Z}^{n}$ on $\overline{\mathbb{Z}}^{n}$ (trivial on the points at infinity) and restrict the action groupoid to $\overline{\mathbb{N}}^{n}$ :

$$
\mathcal{T}_{n}=\mathbb{Z}^{n} \times\left.\overline{\mathbb{Z}}^{n}\right|_{\overline{\mathbb{N}}^{n}}=\left\{(j ; k) \in \mathbb{Z}^{n} \times \overline{\mathbb{Z}}^{n} \mid k_{i}, j_{i}+k_{i} \geq 0\right\}
$$

Consider then the subgroupoid $\tilde{\mathcal{T}}_{n} \subset \mathcal{T}_{n}$ given by:

$$
\tilde{\mathcal{T}}_{n}=\left\{(j ; k) \in \mathcal{T}_{n} \mid k_{i}=\infty \Longrightarrow \sum_{k=1}^{i} j_{k}=0, j_{l}=0, l \geq i+1\right\}
$$

Finally let $\mathfrak{T}_{n}$ be the quotient $\tilde{\mathcal{T}}_{n} / \sim$ where $(j ; k) \sim\left(j ; k_{1}, \ldots, k_{i-1}, \infty, \ldots, \infty\right)$ if $k_{i}=\infty$. We denote with $(j ; k)^{\sim}$ the equivalence class. It is shown in Theorem 1 of [25] that $C\left(\mathbb{C} P_{n, q, 0}\right) \simeq C^{*}\left(\mathfrak{T}_{n}\right)$. The choice of this particular description of the groupoid $C^{*}$-algebra depends on a representation-theoretic construction of $C\left(\mathbb{C} P_{n, q, 0}\right)$ which could be seen as an analogue of the symplectic foliation description in terms of Lu's coordinates (see Appendix (A)).

Proposition 7.1. The map $\Phi: \mathcal{G}_{F}^{b s}\left(\mathbb{C P}_{n}, \pi_{0}\right) \rightarrow \mathfrak{T}_{n}$, defined as

$$
\Phi(c, h)=\left(p_{1}, p_{2}-p_{1}, \ldots ; q_{1}, q_{2}-q_{1}, \ldots\right)^{\sim}, \quad c_{i}=1-e^{-\hbar q_{i}}, h_{i}=\hbar p_{i},
$$

is an isomorphism of topological groupoids.

Proof. The map is easily seen to be well defined once one recalls the range of $(c, h)$ described in Proposition 6.1 and the characterization of Bohr-Sommerfeld leaves given in Theorem 6.3. Remark that in the definition of the map $\Phi$ there is no need of specifying the definition of $q_{i}-q_{i-1}=\infty-\infty$ for $i \geq r+1$, when $c_{r-1}<c_{r}=1$. Indeed, since $q_{r}-q_{r-1}$ is well defined and equal to $\infty$, these entries are not relevant in the equivalence class $\Phi(c, h)$.

It is an easy check to verify that $\Phi$ is a continuous groupoid morphism. The inverse is given by

$$
\Phi^{-1}(j ; k)^{\sim}=(c, h), \quad c_{i}=1-e^{-\hbar \sum_{l=1}^{i} k_{l}}, h_{i}=\hbar \sum_{l=1}^{i} j_{l},
$$

that is well defined and continuous.

Let us also remark that the functorial quantization of Poisson submanifolds $P_{1}(0), \ldots, P_{n}(0)$, in this case, exactly gives rise to the composition sequence described in Corollary 3 of [25], as a simple checking shows. 


\subsection{The non standard $\mathbb{C} P_{1, q, t}$, for $t \in(0,1)$}

Let us now move to the non standard case. In such case Sheu produced an explicit groupoid description only for $n=1$. In the $n>1$ case, the non standard quantum complex projective space is described just as a sub $C^{*}$-algebra of a groupoid $C^{*}$-algebra.

For $n=1$ in 25 ] it was proved that $C\left(\mathbb{C} P_{1, q, t}\right) \simeq C(\mathfrak{G}), t \in(0,1)$, where $\mathfrak{G}$ is the subgroupoid of the action groupoid $\mathbb{Z}^{2} \times\left.\overline{\mathbb{Z}}^{2}\right|_{\overline{\mathbb{N}}^{2}}$

$$
\mathfrak{G}=\left\{\left(j, j, k_{1}, k_{2}\right) \in \mathbb{Z}^{2} \times\left.\overline{\mathbb{Z}}^{2}\right|_{\overline{\mathbb{N}}^{2}} \mid k_{1} \text { or } k_{2}=\infty\right\} .
$$

Let us recall that $\mathcal{G}_{F}^{b s}\left(\mathbb{C} P_{1}, \pi_{t}\right)$ has three components

$$
\begin{aligned}
& \mathcal{G}_{F}^{b s}\left(\mathbb{C} P_{1}, \pi_{t}\right)^{(1,0)}=\left\{(q, p) \in \overline{\mathbb{Z}} \times \mathbb{Z} \mid q \geq q_{t}, q+p \geq q_{t}\right\} \\
& \mathcal{G}_{F}^{b s}\left(\mathbb{C} P_{1}, \pi_{t}\right)^{(0,1)}=\left\{(q, p) \in \overline{\mathbb{Z}} \times \mathbb{Z} \mid q \geq \tilde{q}_{t}, q+p \geq \tilde{q}_{t}\right\} \\
& \mathcal{G}_{F}^{b s}\left(\mathbb{C} P_{1}, \pi_{t}\right)^{(0,0)}=\{(\infty, p) \in \overline{\mathbb{Z}} \times \mathbb{Z}\}
\end{aligned}
$$

where $q_{t}=-\frac{1}{\hbar} \ln (1-t)$ and $\tilde{q}_{t}=-\frac{1}{\hbar} \ln t$. A direct computation shows that:

Proposition 7.2. The map $\Phi: \mathcal{G}_{F}^{b s}\left(\mathbb{C} P_{1}, \pi_{t}\right) \rightarrow \mathfrak{G}$ defined on each component by:

$$
\Phi(q, p)=\left\{\begin{array}{cl}
\left(p, p, \infty, q-q_{t}\right) & \text { if }(q, p) \in \mathcal{G}_{F}^{b s}\left(\mathbb{C} P_{1}, \pi_{t}\right)^{(1,0)} \\
\left(p, p, q-\tilde{q}_{t}, \infty\right) & \text { if }(q, p) \in \mathcal{G}_{F}^{b s}\left(\mathbb{C} P_{1}, \pi_{t}\right)^{(0,1)} \\
(p, p, \infty, \infty) & \text { if }(q, p) \in \mathcal{G}_{F}^{b s}\left(\mathbb{C} P_{1}, \pi_{t}\right)^{(0,0)}
\end{array}\right.
$$

is an isomorphism of topological groupoids for all $t \in(0,1)$.

The existence of this isomorphism also proves that the groupoids of BS-leaves and so the groupoid $C^{*}$-algebras are not depending on $t$, as long as $0<t<1$.

\subsection{Odd dimensional quantum spheres $\mathbb{S}_{q}^{2 n-1}$}

From the discussion in Section 4 we know that the Poisson submanifold $P_{n}(t) \subset$ $\left(\mathbb{C} P_{n}, \pi_{t}\right)$ is isomorphic to the odd Poisson sphere $\mathbb{S}^{2 n-1}$. We want to compare the groupoid $\mathcal{G}_{F}^{b s}\left(P_{n}(t), \pi_{t}\right)$ appearing in Proposition 6.4 with the characterization of the quantum sphere given in [26], where it is shown that $C\left(\mathbb{S}_{q}^{2 n-1}\right) \simeq C^{*}\left(\mathfrak{F}_{n-1}\right)$, where $\mathfrak{F}_{n-1}$ is a groupoid that we are going to describe.

Let $\mathcal{F}_{n-1}$ be the following subgroupoid of the action groupoid (with the first $\mathbb{Z}$ acting trivially)

$$
\left\{(z ; x ; w) \in \mathbb{Z} \times\left.\left(\mathbb{Z}^{n-1} \times \overline{\mathbb{Z}}^{n-1}\right)\right|_{\overline{\mathbb{N}}^{n-1}} \mid w_{i}=\infty \Rightarrow z=-\sum_{j} x_{j}, x_{k}=0, k>i\right\}
$$

Define on it the equivalence relation $(z ; x ; w) \sim\left(z ; x ; w_{1}, \ldots, w_{i}, \infty\right)$ if $w_{i}=\infty$ and let $\mathfrak{F}_{n-1}=\mathcal{F}_{n-1} / \sim$. Let us denote with $(z ; x ; w)^{\sim} \in \mathfrak{F}_{n-1}$ the equivalence class. Let, as before, $q_{t}=-\frac{1}{\hbar} \log (1-t)$. 
Proposition 7.3. The following map $\Phi: \mathcal{G}_{F}^{b s}\left(P_{n}(t), \pi_{t}\right) \rightarrow \mathfrak{F}_{n-1}$ defined as follows

$$
\Phi(c, h)=\left(-p_{n} ; p_{1}, p_{2}-p_{1}, \ldots, p_{n-1}-p_{n-2} ; q_{1}-q_{t}, q_{2}-q_{1}, \ldots, q_{n-1}-q_{n-2}\right)^{\sim},
$$

where $h_{k}=\hbar p_{k}$ and $c_{k}=1-t-e^{-\hbar q_{k}}$ for $k<n$, is an isomorphism of topological groupoids.

Proof. Let us recall that if $(c, h) \in \mathcal{G}_{F}^{b s}\left(P_{n}(t), \pi_{t}\right)$ then $c_{n}=1-t$. If $c_{i-1}<$ $c_{i}=1-t$, for some $i$ then $w_{i}=q_{i}-q_{i-1}=\infty$ is well defined and is not relevant how we define $w_{r}=q_{r}-q_{r-1}=\infty-\infty$ for $r>i$ because of the equivalence relation. Furthermore, in this case $x_{k}=p_{k}-p_{k-1}=0$ for $k>i$ and $\sum_{j} x_{k}=p_{i}=p_{i+1}=\ldots=p_{n}=-z$. The inverse $\Phi^{-1}: \mathfrak{F}_{n-1} \rightarrow \mathcal{G}_{F}^{b s}\left(\mathbb{C} P_{n}, \pi_{t}\right)$ is given by

$$
c_{i}=(1-t)\left(1-e^{-\hbar \sum_{j=1}^{i} w_{j}}\right), h_{i}=\hbar \sum_{j=1}^{i} x_{j}, c_{n}=1-t, h_{n}=-\hbar z \quad .
$$

The proof that $\Phi$ is a groupoid morphism is straightforward.

Since the functor assigning to a groupoid its $C^{*}$-algebra is contravariant there is a $C^{*}$-algebra epimorphism from the $C^{*}$-algebra of $\mathbb{C} P_{n, q, t}$, for $t \in(0,1)$, to the $C^{*}$-algebra of the odd-dimensional quantum sphere $\mathbb{S}_{q}^{2 n-1}$. This is exactly the analogue of Theorem 6 in [25].

\section{Conclusions}

Quantum homogeneous spaces as groupoid $C^{*}$-algebras. In Theorem 6.3 we proved that the groupoid of Bohr-Sommerfeld leaves $\mathcal{G}_{F}^{b s}\left(\mathbb{C} P_{n}, \pi_{t}\right)$ has a unique Haar system for all $t \in[0,1]$. According to our program, we can define the convolution algebra and the $C^{*}$-algebra out of these data. For $t=0$, by using Sheu's results we know that this is exactly the $C^{*}$-algebra defined from the quantum homogeneous space $\mathbb{C} P_{n, q, 0}$. In the case $t \in(0,1)$ Sheu identifies the quantum algebra only as a subalgebra of a groupoid $C^{*}$-algebra. It would be very interesting to have a proof based on the representation theory of $\mathbb{C} P_{n, q, t}$ that its $C^{*}$-algebra $C\left(\mathbb{C} P_{n, q, t}\right)$ is isomorphic to $C^{*}\left(\mathcal{G}_{F}^{b s}\left(\mathbb{C} P_{n}, \pi_{t}\right)\right)$ also for $t \in(0,1)$. Analogously it would be interesting to determine the polynomial algebra of quantum $P_{k}(t)$ and show that its $C^{*}$-algebra is isomorphic to $C^{*}\left(\mathcal{G}_{F}\left(P_{k}(t), \pi_{t}\right)\right.$ ) (we discussed the case $k=1, n$ in Subsection (7.3).

Compact Hermitian symmetric spaces. The basic ingredient of the construction that we have presented in this paper is the bihamiltonian system on $\mathbb{C} P_{n}$. This 
system exists for all compact hermitian symmetric spaces; most of the results extend to the general case. In particular, the proof of the multiplicativity of the modular function is general; given the eigenvalues of the Nijenhuis operator, the structure maps of the groupoid of leaves are expressed by the same formulas.

While the existence of the bihamiltonian system is known since long time, at the best of our knowledge, $\mathbb{C} P_{n}$ is the only case that has been investigated. The key characterization that we need is the diagonalization of the Nijenhuis operator: for $\mathbb{C} P_{n}$ is a result easy to obtain (Proposition 5.1). In the general case we don't know if global eigenvalues of the Nijenhuis operator exist, how to describe them and finally if the whole quantization procedure works. This requires further investigation, that makes a reasonable plan for our future research.

Prequantization cocycle. The prequantization cocycle did not appear in our final quantization setting; this means that we produce our quantum algebras by taking it to be trivial. On one side, in those cases where we can compare our results with quantum group constructions, via Sheu's results, there is no need of a non trivial 2-cocycle. On the other side we know that, at least in some examples but we expect it in general, its cohomology class is not trivial. It is possible that it makes a difference what kind of cohomology we consider, differentiable or just continuous, and that for the $C^{*}$-algebras smooth properties are not relevant. An indication in this direction is given by recent results of [28] where it is shown that certain Poisson-homogeneous structures which are cohomologically non trivial may become trivial if one admits vector fields which are smooth along the symplectic leaves and globally only continuous. In any case, the role of this prequantization cocycle is still unclear.

Morita equivalences. The Poisson structures $\left(\mathbb{C} P_{n}, \pi_{t}\right)$ are not in general Poisson diffeomorphic for different $t$. In [4, 23] it was proven that non standard $\mathbb{C} P_{1}$ Poisson structures are non diffeomorphic for different values of $t$. In fact the Poisson invariant allowing to distinguish them is the so called regolarized Liouville volume $V(\pi)=\ln \frac{1-t}{1+t}$. However, since the modular periods are the same, apart from the singular $t=0,1$ case, non standard Poisson $\mathbb{C} P_{1}$ are all Poisson-Morita equivalent. The fact that the corresponding groupoid of Bohr-Sommerfeld leaves does not depend, as topological groupoid, on $t$ can be seen as a quantum analogue of this fact. It would be interesting to analyze these properties for higher $n$. We remark that the example developed in [3] shows that even non PoissonMorita equivalent structures can produce the same topological groupoid of BS leaves. We expect that, like in the $n=1$ case, all $\left(\mathbb{C} P_{n}, \pi_{t}\right)$ are at least Morita equivalent. We plan to investigate this equivalence and how it is quantized to the Bohr-Sommerfeld groupoids.

Acknowledgments The research of J.Q. is supported by the Luxembourg FNR grant PDR 2011-2, and by the UL Grant GeoAlgPhys 2011-2013 


\section{A Review of Lu's Coordinates}

In this section, we recall the derivation of Lu's parametrization of Bruhat cells [17]. Do notice that, in contrast to the main text, the homogeneous spaces such as the flag manifolds are presented as right cosets, e.g. $\mathbb{C} P_{n}=S U(n+1) / U(n)$. This is to avoid certain awkwardness in the presentation, no conclusions in the main text will be changed.

We focus on the case $K=S U(n), K^{*}=S B(n)$ and $G=K_{\mathbb{C}}=S L(n, \mathbb{C})$, for such groups, the concepts such as the Bruhat Poisson structure, Bruhat cells, dressing actions, etc can all be understood rather concretely.

Our focus is the flag manifold flag $\left(n_{1}, \cdots, n_{k}\right)$, where the string of increasing integers $0=n_{0}<n_{1}<n_{2}<\cdots<n_{k}<n_{k+1}=n$ denotes the nested subspaces of $\mathbb{C}^{n}$ of dimension $n_{1}, n_{2}, \cdots, n_{k}$. A point in the flag can be represented by a matrix $g \in S L(n)$, suppose $g=\left[v_{1}, \cdots v_{n}\right]$, where $v_{i}$ are column vectors, then the flag in question is $\operatorname{span}\left(v_{1}, \cdots v_{n_{1}}\right) \subset \operatorname{span}\left(v_{1}, \cdots v_{n_{2}}\right) \subset \operatorname{span}\left(v_{1}, \cdots v_{n_{k}}\right) \subset \mathbb{C}^{n}$. There are certain redundancies of such a parametrization, for example, a right multiplication by a matrix in $S B$ does not change the flag, since these matrices are upper-triangular. In general, unless $n_{i}-n_{i-1}=1$ for all $1 \leq i \leq k$, i.e. the full-flag manifold, the stability group is bigger than $S B$.

In contrast, the left multiplication by $\gamma \in S B$ on $g=\left[v_{1}, \cdots v_{n}\right] \in S U$ induces a non-trivial action on the flag, so one can decompose the flag manifold into orbits of this action. The action is related to the dressing action reviewed in the text, write $\gamma \cdot g={ }^{\gamma} g \cdot \gamma^{g}$, with ${ }^{\gamma} g \in S U$ and $\gamma^{g} \in S B$. One can discard $\gamma^{g}$ since ${ }^{\gamma} g \cdot \gamma^{g}$ and ${ }^{\gamma} g$ give the same flag. If the stability group were exactly $S B$, then the action can be identified with the dressing transformation.

To cut down the stability group one can do the following. The identity matrix corresponds to $\left[e_{1}, \cdots, e_{n}\right]$, where $e_{i}$ are the standard basis vectors, while an element of the Weyl group $W$, when represented as a matrix, corresponds to $\left[e_{\sigma(1)}, \cdots, e_{\sigma(n)}\right]$, where $\sigma$ is a permutation (recall that the Weyl group for $S U$ are none other than permutations). One can certainly reach any point in the flag by left multiplying $S B$ to a suitable $w \in W$, thus one can label the orbits of the left $S B$ action by elements of $W$. Further introduce a subset of permutations called shuffles, denoted as $\sigma_{n}\left(n_{1}, n_{2}, \cdots, n_{k}\right)$, consisting of $\left\{\sigma \in S_{n} \mid \sigma(i)<\sigma(j)\right.$ if $n_{k}<$ $\left.i<j \leq n_{k+1}\right\}$. It is clear that the shuffles $\sigma\left(n_{1}, \cdots, n_{k}\right)$ label all the orbits of left $S B$ action in the flag, since permutations within each subspace spanned by, say, $\left\{e_{n_{i}+1}, \cdots e_{n_{i+1}}\right\}$ does not change the flag. The point is that now one may impose a 'gauge condition' on the representative of a point in the flag, as follows. One defines an ordering on the vectors $\left\{v_{i}\right\}$ that span the flag by saying that $v<v^{\prime}$ if the last nonzero component of $v^{\prime}$ appears later than that of $v$. The gauge condition is such that: if $n_{k}<i<j \leq n_{k+1}$, then $v_{i}<v_{j}$. It is clear that the image of the left $S B$ action on a shuffle satisfies this gauge condition. 
Once this condition is enforced, the stability group is cut down to being the right multiplication by $S B$. To summarize, the flag has the following decomposition

$$
\operatorname{flag}\left(n_{1} \cdots, n_{k}\right)=\bigcup_{w} S B w S B / S B
$$

where the summation is over $\sigma_{n}\left(n_{1}, n_{2}, \cdots, n_{k}\right)$-shuffles.

Next, we shall identify a subgroup $N_{w} \subset S B$ whose left action on a particular shuffle $w$ is free. Let $\xi \in \mathfrak{s b}$, then the action of $\xi$ on $w$ is nonzero if $p_{1} A d_{w^{-1}} \xi \neq 0$, where $p_{1}: \mathfrak{s l} \rightarrow \mathfrak{s u}$ is the projection. Recall that $S B$ is a subgroup generated by the positive roots of $\mathfrak{s u}$ and $i \mathfrak{t}$, where $\mathfrak{t}$ is the Cartan. One sees that for a given $w$, the subset of roots $\Phi_{w}=\left\{\alpha>0 \mid A d_{w^{-1}} \alpha<0\right\}$ will generate a nonzero action since $p_{1} A d_{w^{-1}} \alpha \neq 0$, and thus $N_{w}$ is generated by $\Phi_{w}$.

One can always decompose an element $w$ of the Weyl group into a string of Weyl reflections $w=w_{\alpha_{k}} \cdots w_{\alpha_{1}}$, where each $\alpha_{i}$ is a simple root and $w_{\alpha_{i}}$ is the Weyl reflection of $\alpha_{i}$. If we denote by $C_{w}$ the image of the left $S B$ action on $w$, then $\mathrm{Lu}$ showed that there is a diffeomorphism

$$
C_{\alpha_{k}} \times \cdots \times C_{\alpha_{1}} \rightarrow C_{w}
$$

where we have abbreviated $C_{w_{\alpha_{i}}}$ as $C_{\alpha_{i}}$. The map is given by taking the product of the lhs, furthermore, this map is a Poisson diffeomorphism, where the lhs is given the product Poisson structure. This gives us a very practical way of parametrizing the orbit $C_{w}$ by that of $C_{\alpha}$. Recall that a simple root $\alpha$ gives an embedding of $S U(2)$ into $S U(n)$, so we may focus on the $S U(2)$ case.

For $S U(2)$, the non-trivial Weyl group element can be chosen as $w=i \sigma_{2}$, where $\sigma_{i}$ are Pauli matrices. There is only one simple root $\sigma_{+}=\left(\sigma_{1}+i \sigma_{2}\right) / 2$, and $w^{-1} \sigma_{+} w=-\sigma_{-}$, so $N_{w}$ is generated by $\sigma_{+}$, and hence can be parametrized as

$$
\gamma(y)=e^{y \sigma_{+}}=\left(\begin{array}{ll}
1 & y \\
0 & 1
\end{array}\right), \quad y \in \mathbb{C}
$$

it is then easy to work out the orbit of the dressing action

$$
\gamma w=\epsilon^{-1}\left[\begin{array}{cc}
-y & 1 \\
-1 & -\bar{y}
\end{array}\right]\left[\begin{array}{cc}
\epsilon & 0 \\
0 & \epsilon^{-1}
\end{array}\right]\left[\begin{array}{cc}
1 & -\epsilon^{-2} \bar{y} \\
0 & 1
\end{array}\right], \quad \epsilon=\left(1+|y|^{2}\right)^{1 / 2}
$$

where the last two factors belong to $S B$. Thus the orbit of the dressing action is parametrized as

$$
g(y)=\frac{1}{\epsilon}\left[\begin{array}{cc}
-y & 1 \\
-1 & -\bar{y}
\end{array}\right], \quad \epsilon=\left(1+|y|^{2}\right)^{1 / 2} .
$$

This orbit actually covers all points of the flag $\mathbb{C} P_{1}$ except the point $z=\infty$, i.e. the unique dimension 2 Schubert cell of $\mathbb{C} P_{1}$. 
To work out the general formula Eq 30 for $\mathbb{C} P_{n}$, we fix the simple roots for $S U(n+1)$ to be: $\alpha_{i}=[0, \cdots \underbrace{1,-1}_{i, i+1}, 0, \cdots 0]$, then the Weyl reflection $w_{\alpha_{i}}$ is an identity matrix except that the $(i, i+1)$ diagonal block is occupied by $i \sigma_{2}$. Next we work out as an example the parametrization of the largest cell of $\mathbb{C} P_{n}$. The Weyl group element corresponding to this cell is the shuffle $\sigma_{n+1}(1)$, and it can be decomposed into

$$
\sigma_{n+1}(1)=w_{\alpha_{n}} \cdots w_{\alpha_{1}}
$$

The orbit of each $C_{\alpha_{i}}$ is parametrized as a rank $(n+1)$ block diagonal matrix,

$$
\mathrm{g}_{i}\left(y_{i}\right)=\left[\begin{array}{ccc}
\mathbf{1}_{i-1} & 0 & 0 \\
0 & g\left(y_{i}\right) & 0 \\
0 & 0 & \mathbf{1}_{n-i}
\end{array}\right]
$$

where $g(y)$ is defined in Eq, 31 . It is then not hard to compute the product Eq 30 explicitly, in fact, we will only need the first column of $C_{w}$, which is given by

$$
\begin{aligned}
& {\left[\mathrm{g}_{n}\left(y_{n}\right) \cdots \mathrm{g}_{1}\left(y_{1}\right)\right]_{\bullet, 1}} \\
& =\frac{1}{\epsilon_{1} \cdots \epsilon_{n}}\left[(-1)^{n} y_{1} \epsilon_{2} \cdots \epsilon_{n}, \quad \cdots \quad-y_{n-2} \epsilon_{n-1} \epsilon_{n}, \quad y_{n-1} \epsilon_{n}, \quad-y_{n}, \quad-1\right]^{T} \text {, }
\end{aligned}
$$

where $\epsilon_{i}=\left(1+\left|y_{i}\right|\right)^{1 / 2}$. This first column of $C_{w}$ should be identified with the homogeneous coordinates $\left[X_{1}, \cdots X_{n+1}\right]$ of $\mathbb{C} P_{n}$, and $C_{w}$ corresponds to the cell where $X_{n+1} \neq 0$. For convenience of the identification, we will make a redefinition of variables $y_{n-1} \rightarrow-y_{n-1}, y_{n-3} \rightarrow-y_{n-3}, \cdots$, then we have

$$
z_{n}=\frac{X_{n}}{X_{n+1}}=y_{n}, \quad z_{n-1}=\frac{X_{n-1}}{X_{n+1}}=y_{n-1} \epsilon_{n}, \cdots .
$$

And we have the inverse map

$$
\begin{aligned}
y_{n} & =z_{n} \\
y_{n-1} & =z_{n-1}\left(1+\left|z_{n}\right|^{2}\right)^{-1 / 2} \\
y_{n-2} & =z_{n-2}\left(1+\left|z_{n-1}\right|^{2}+\left|z_{n}\right|^{2}\right)^{-1 / 2} \\
& \cdots \\
y_{1} & =z_{1}\left(1+\left|z_{2}\right|^{2}+\cdots+\left|z_{n}\right|^{2}\right)^{-1 / 2} .
\end{aligned}
$$

These are the Lu's coordinates quoted in (10). They can be thought as coordinates on leaves of different copies of $S U(2)$ projected onto the complex projective space. Let us remark that in principle all that is contained in here applies also to the nonstandard case, the only difference being in the projection map $p_{t}$, which will pick a $t$-depending linear combination of the first and last column of $C_{w}$. 


\section{A.1 The Kirillov-Kostant symplectic form}

In the text, we shall also need the symplectic Kirillov-Kostant symplectic form for $\mathbb{C} P_{n}$, we review here the construction of this form and some concrete formulae and show that it takes a very simple form in Lu's coordinates.

For a Lie group $K$, the coadjoint orbit associated with $\lambda \in \mathfrak{g}^{*}$ is $\mathcal{O}_{\lambda}=$ $\left\{\left(A d_{g}^{-1}\right)^{*} \lambda \mid g \in K\right\}$, and it is diffeomorphic to $K / H_{\lambda}$ where $H_{\lambda}$ is the stability group of $\lambda$. Over $\mathcal{O}_{\lambda}$, one can write down a form

$$
\omega_{\lambda}=i d \operatorname{Tr}\left[g^{-1} d g \lambda\right]=-i \operatorname{Tr}\left[\left(g^{-1} d g\right)^{2} \lambda\right],
$$

It is non-degenerate and closed (but not exact).

In the case of $K=S U(n+1)$, the coadjoint orbit passing through

$$
\lambda=\frac{1}{n+1} \operatorname{diag}[n, \underbrace{-1, \cdots,-1}_{n}]
$$

is nothing but $\mathbb{C} P_{n}$, since $H_{\lambda}$ in this case is clearly $S(U(1) \times U(n))$.

For $S U(2)$ the computation is simple, letting $\lambda=[1 / 2,-1 / 2]$ and parametrize a group element as $g(y) e^{i \theta \sigma_{3}}$ (where $g(y)$ is defined in Eq31), one gets

$$
\omega_{\lambda}=\frac{i}{2} d \operatorname{Tr}\left[g^{-1} d g \sigma_{3}\right]=\frac{-i d y \wedge d \bar{y}}{\left(1+|y|^{2}\right)^{2}},
$$

the Fubini-Study Kähler form on $\mathbb{C} P_{1}$.

To obtain the Kirillov-Kostant for $\mathbb{C} P_{n}$, we take $\lambda$ as in Eq.36, i.e. the highest weight of the fundamental representation of $S U(n+1)$. Then we shall use the Lu parametrization for $S U(n+1) / S(U(1) \times U(n))$. The largest cell in this quotient is labeled by the shuffle $w=\sigma_{n+1}(1)$, and it can be decomposed as in Eq.32 The cell $C_{w}$ is now a product

$$
C_{w}=C_{\alpha_{n}} \cdots C_{\alpha_{2}} C_{\alpha_{1}},
$$

We parametrize this product $C_{\alpha_{n}} \cdots C_{\alpha_{2}} C_{\alpha_{1}}$ as $\mathrm{g}_{n}\left(y_{n}\right) \cdots \mathrm{g}_{1}\left(y_{1}\right)$, where $\mathrm{g}_{i}\left(y_{i}\right)$ is defined in Eq 33 ,

One may now proceed to compute the Liouville form $\operatorname{Tr}\left[g^{-1} d g \lambda\right]$ leading to

$$
\begin{gathered}
\operatorname{Tr}\left[g^{-1} d g \lambda\right]=i A\left(z_{1}\right)+\frac{i}{\epsilon_{1}^{2}} A\left(y_{2}\right)+\frac{i}{\epsilon_{1}^{2} \epsilon_{2}^{2}} A\left(y_{3}\right)+\cdots \frac{i}{\epsilon_{1}^{2} \cdots \epsilon_{n-1}^{2}} A\left(y_{n}\right), \\
A(y)=\frac{i}{2} \frac{y d \bar{y}-y d \bar{y}}{\left(1+|y|^{2}\right)} .
\end{gathered}
$$

Let us plug in the change of variables Eq 34 and get the neat result

$$
\operatorname{Tr}\left[g^{-1} d g \lambda\right]=-\frac{1}{2} \frac{\sum_{i=1}^{n} z_{i} d \bar{z}_{i}-c . c}{1+\sum_{i=1}^{n}\left|z_{i}\right|^{2}} .
$$

The differential of the above 1-form is recognized as the Fubini-Study Kähler form for $\mathbb{C} P_{n}$. 


\section{A.2 Concrete formulae for the Bruhat Poisson structure}

In this section we collect some formulae for the Poisson structure of $S U(2)$ and its dual group $S B(2)$, as well as the symplectic form on the double $S L(2)$. The computation leading to these results are quite lengthy and is omitted.

Parametrize an element of $S U(2)$ as $g(y) e^{i \theta \sigma_{3}}$, and plug it into Eq,6, a straightforward calculation shows

$$
\pi_{S U(2)}=i\left(1+|y|^{2}\right) \frac{\partial}{\partial y} \wedge \frac{\partial}{\partial \bar{y}}
$$

which vanishes when $y \rightarrow \infty$. As mentioned above the map Eq 30 is a Poisson map, so the Poisson tensor for a general cell is just the sum of the results above.

The group $S B(2)$ is dual group of $S U(2)$, and has a simple parametrization $\gamma=e^{\phi \sigma_{3}} e^{u \sigma_{+}}$, and the dual Poisson structure in this coordinate system is

$$
-2 \pi_{S B(2)}=2 i\left(-\left(1+|u|^{2}\right)+e^{-4 \phi}\right) \partial_{u} \wedge \partial_{\bar{u}}+i u \partial_{u} \wedge \partial_{\phi}-i \bar{u} \partial_{\bar{u}} \wedge \partial_{\phi} .
$$

On the double $S L(2)$, one has a Poisson structure $\Pi$ defined in Eq2, which is non-degenerate, so its inverse defines a symplectic structure. If one parametrizes the double as $\gamma \cdot g=e^{\phi \sigma_{3}} e^{u \sigma_{+}} g(y) e^{i \theta \sigma_{3}}$, then

$$
\Pi^{-1}=\frac{i}{\left(1+|w|^{2}\right)} d w d \bar{w}-\frac{i}{\left(1+|y|^{2}\right)} d y d \bar{y}-2 d \theta d \log \frac{\epsilon^{2}(w)}{e^{2 \phi} \epsilon^{2}(y)},
$$

where $w=e^{2 \phi}(u+y)$. This nice structure of the symplectic form persists for a more general group, and the result and proof thereof shall appear in a forthcoming publication.

\section{B An alternative proof of Lemma 5.1}

We also present a proof using direct computation so as to be self-containing. Denote $U_{0}=S(U(1) \times U(n)) \subset S U(n+1)$, and $U_{t}=A d_{\sigma_{t}} U_{0}$. Now $U_{0}$ is a Poisson-Lie subgroup while $U_{t}$ is coisotropic. The quotient $U_{t} \backslash S U(n+1)$ is also isomorphic to $\mathbb{C} P_{n}$, and there is a well-defined map between the cosets $\phi_{t}: U_{t} \backslash S U(n+1) \rightarrow U_{0} \backslash S U(n+1)$,

$$
[g]_{t} \stackrel{\phi_{t}}{\rightarrow}\left[\sigma_{t}^{-1} g\right]_{0}, \quad g \in S U(n+1)
$$

where []$_{t}$ is the quotient w.r.t $U_{t}$.

Since $U_{t}$ is coisotropic, there is a Poisson structure $\pi_{t}$ on $U_{t} \backslash S U(n+1)$ inherited from that of $S U(n+1)$. Concretely, one chooses locally a representative $g$ 
of $[g]_{t}$, one can then trivialize $T^{*}\left(U_{t} \backslash S U(n+1)\right)$ by the right invariant 1-forms: $r_{g^{-1}}^{*} \xi, \xi \in \mathfrak{u}_{t}^{\perp}$, and under a change of representative $g \rightarrow h g, h \in U_{t}$, one has $\xi \rightarrow A d_{h^{-1}}^{*} \xi$. One then defines

$$
\pi_{t}\left([g]_{t}\right)\left(r_{g^{-1}}^{*} \xi, r_{g^{-1}}^{*} \eta\right)=\pi(g)\left(r_{g^{-1}}^{*} \xi, r_{g^{-1}}^{*} \eta\right),
$$

where $\pi$ is the Poisson tensor of $S U(n+1)$ and one can check that this definition is independent of the choice of the local representative, thanks to the multiplicativity of $\pi$.

Next, we will push $\pi_{t}$ forward to $U_{0} \backslash S U(n+1)$ using the map (40). Let now $\xi, \eta \in \mathfrak{u}_{0}^{\perp}$, and plug the 1-forms $r_{g^{-1}}^{*} \xi, r_{g^{-1}}^{*} \eta$ into $\phi_{t *} \pi_{t}$,

$$
\begin{aligned}
& \left(\phi_{t *} \pi_{t}\right)(g)\left(\left(r_{g^{-1}}\right)^{*} \xi,\left(r_{g^{-1}}\right)^{*} \eta\right)=\pi\left(\sigma_{t} g\right)\left(\left(l_{\sigma_{t}^{-1}}\right)^{*}\left(r_{g^{-1}}\right)^{*} \xi,\left(l_{\sigma_{t}^{-1}}\right)^{*}\left(r_{g^{-1}}\right)^{*} \eta\right) \\
= & \left(l_{\sigma_{t} *} \pi(g)+r_{g^{*}} \pi\left(\sigma_{t}\right)\right)\left(\left(l_{\sigma_{t}^{-1}}\right)^{*}\left(r_{g^{-1}}\right)^{*} \xi,\left(l_{\sigma_{t}^{-1}}\right)^{*}\left(r_{g^{-1}}\right)^{*} \eta\right) \\
= & \pi(g)\left(\left(r_{g^{-1}}\right)^{*} \xi,\left(r_{g^{-1}}\right)^{*} \eta\right)+\pi\left(\sigma_{t}\right)\left(\left(l_{\sigma_{t}^{-1}}\right)^{*} \xi,\left(l_{\sigma_{t}^{-1}}\right)^{*} \eta\right) \\
= & \pi(g)\left(\left(r_{g^{-1}}\right)^{*} \xi,\left(r_{g^{-1}}\right)^{*} \eta\right)-\pi\left(\sigma_{t}^{-1}\right)\left(\left(r_{\sigma_{t}}\right)^{*} \xi,\left(r_{\sigma_{t}}\right)^{*} \eta\right)
\end{aligned}
$$

where in the last step we used the multiplicativity of $\pi$ and that $\pi(1)=0$.

The first term of (41) gives $\pi_{0}$, we want to show that the second term gives $\pi_{\lambda}$, namely it is the inverse of the Kirillov-Kostant symplectic form

$$
\omega_{\lambda}=-i \operatorname{Tr}\left[\left(d g \cdot g^{-1}\right)^{2} \lambda\right], \quad \lambda=(n+1)^{-1} \operatorname{diag}[n, \underbrace{-1, \cdots,-1}_{n}],
$$

note the change of convention regarding left and right invariance compared to the formula (35) in Appendix Apply the formula (6) for $\pi$,

$$
-\pi\left(\sigma_{t}^{-1}\right)\left(\left(r_{\sigma_{t}}\right)^{*} \xi,\left(r_{\sigma_{t}}\right)^{*} \eta\right)=-\frac{1}{2}\left\langle p_{1} A d_{\sigma_{t}} \xi, p_{2} A d_{\sigma_{t}} \eta\right\rangle .
$$

Now let

$$
\xi=\left(\begin{array}{cc}
0 & \vec{v} \\
0 & 0
\end{array}\right), \quad \eta=\left(\begin{array}{cc}
0 & \vec{w} \\
0 & 0
\end{array}\right), \quad \xi, \eta \in \mathfrak{u}_{0}^{\perp}
$$

A direct calculation shows

$$
\left\langle p_{1} \sigma_{t} \xi \sigma_{t}^{-1}, p_{2} \sigma_{t} \eta \sigma_{t}^{-1}\right\rangle=-t \operatorname{Im}\left(\vec{v} \cdot \vec{w}^{\dagger}\right),
$$

thus $-\pi\left(\sigma_{t}^{-1}\right)\left(\left(r_{\sigma_{t}}\right)^{*} \xi,\left(r_{\sigma_{t}}\right)^{*} \eta\right)=t / 2 \operatorname{Im}\left(\vec{v} \cdot \vec{w}^{\dagger}\right)$.

Next, let $X, Y \in \mathfrak{s u}(n+1)$ and evaluate (42) on $r_{g *} X, r_{g *} Y$,

$$
\omega_{\lambda}\left(r_{g *} X, r_{g *} Y\right)=-i \operatorname{Tr}[[X, Y] \lambda]=2 \sum_{k=2}^{n+1} \operatorname{Im}\left(X_{1 k} Y_{k 1}\right),
$$

comparing this with (43), we conclude

$$
\phi_{t *} \pi_{t}=\pi_{0}+t \omega_{\lambda}^{-1}=\pi_{0}+t \pi_{\lambda}
$$




\section{References}

[1] F. Bonechi, N. Ciccoli, N. Staffolani, M. Tarlini, On the integration of Poisson homogeneous spaces. Journal of Geometry and Physics, 58, (2008) 1519-1529.

[2] F. Bonechi, N. Ciccoli, N. Staffolani, M. Tarlini, The quantization of the symplectic groupoid of the standard Podles sphere. Journal of Geometry and Physics, 62, (2012) 1851-1865.

[3] F. Bonechi, N. Ciccoli, M. Tarlini, The modular class as a quantization invariant. PoS(ICMP 2012)003.

[4] H. Bursztyn, O. Radko, Gauge equivalence of Dirac structures and symplectic groupoids. Ann. Inst. Fourier, 53, (2003) 309-337.

[5] A.S. Cattaneo, G. Felder, Poisson sigma models and symplectic groupoids. In "Quantization of Singular Symplectic Quotients", (ed. N. P. Landsman, M. Pflaum, M. Schlichenmeier), Progress in Mathematics, 198, (2001) $41-73$.

[6] A. S. Cattaneo, G. Felder, L. Tomassini, From local to global deformation quantization of Poisson manifolds. Duke Math.J., 115, (2002) 329-352.

[7] N. Ciccoli, A.J.-L. Sheu, Covariant Poisson Structures on Complex Grassmannians. Comm. Anal. Geom., 14, (2006) 443-474.

[8] M. Crainic, Prequantization and Lie brackets. J. Symplectic Geom., 2, (2004) 579-602.

[9] M. Crainic, R. L. Fernandes, Integrability of Lie brackets. Ann. of Math., 157, (2003) 575-620.

[10] M. Crainic, R.L. Fernandes, Integrability of Poisson Brackets. J. Differential Geom., 66, (2004), 71-137.

[11] P. A. Damianou, R. L. Fernandes, Integrable hierarchies and the modular class. Ann. Inst. Fourier, 58, (2008) 107-137.

[12] M. Dijkhuizen, M. Noumi, A family of quantum projective spaces and related q-hypergeometric orthogonal polynomials. Trans. Amer. Mat. Soc., 350, (1998) 3269-3296.

[13] P. Foth, Bruhat Poisson structure on $\mathbb{C} P_{n}$ and integrable systems. J. Math. Phys., 43, (2002) 3124-3132. 
[14] E. Hawkins, A groupoid approach to quantization. J. Symplectic Geom., 6, (2008) 61-125.

[15] M. Kontsevich, Deformation Quantization of Poisson Manifolds. Lett. Math. Phys., 66, 3, (2003), 157-216.

[16] S. Khoroshkin, A. Radul, V. Rubtsov, A family of Poisson structures on hermitian symmetric spaces. Commun. Math. Phys., 152, 2, (1993), 299-315.

[17] J. -H. Lu, Coordinates on Schubert's cells, Kostant's harmonic forms and the Bruhat Poisson structure on G/B. Transformation Groups Volume 4, Number 4 (1999), 355-374.

[18] J.-H. Lu, A. Weinstein, Groupoides symplectiques doubles de groupes de Lie-Poisson. Compt. Rend. Sci. Ser. I Math., 309, (1989) 951-954.

[19] J.-H. Lu, A. Weinstein, Poisson Lie groups, dressing transformations and Bruhat decomposition. J. Differential Geom., 31, (1990), 501-526.

[20] F. Magri, C. Morosi, A geometrical characterization of Hamiltonian systems through the theory of Poisson-Nijenhuis manifolds. Università di Milano-Bicocca, Quaderni di Matematica n.3/2008.

[21] I. Moerdijk, J. Mrcun, Introduction to Foliations and Lie groupoids. Cambridge University Press, (2003).

[22] J. Renault, A groupoid approach to $C^{*}$-algebras. Lectures Notes in Mathematics 793, Springer Verlag (1980).

[23] O. Radko, A classification of topologically stable Poisson structures on a compact oriented surface. J. Symplectic Geometry, 1, (2002) 523-542.

[24] M. Saito, Sur certaines groupes de Lie resolubles. Sci. Papers Coll. Gen. Educ. Univ. Tokyo , 7, (1957) 1-11.

[25] A.J.-L. Sheu, Groupoid Approach to Quantum Projective Spaces. Contemporary Mathematics, 228, (1998) 341-350

[26] A. J.-L. Sheu, Quantum spheres as groupoid $C^{*}$-algebras. The Quarterly Journal of Mathematics, 48, (1997) 503-510.

[27] A. J.-L. Sheu, Covariant Poisson structures in complex projective spaces. Comm. Anal. Geom., 10, (2002) 61-78. 
[28] A. J.-L. Sheu, Scaling of Poisson spheres and compact Lie groups. Asian J. Math., 16, (2012) 775-786.

[29] J. Stokman, The quantum orbit method for generalized flag manifolds. Math. Res. Lett., 10, (2003) 469-481.

[30] L.L. Vaksman, Ya. S. Soibelman, Algebra of functions on the quantum group $\mathrm{SU}(n+1)$, and odd-dimensional quantum spheres. Leningrad Math. J., 2, (1991) 1023-1042.

[31] A. Weinstein, P. Xu, Extensions of symplectic groupoids and quantization. J. Reine Angew. Math., 417, (1991) 159-189 